\title{
VISCOELASTIC MULTI-MODE SIMULATIONS OF WIRE-COATING
}

\author{
H. Matallah, P. Townsend and M.F. Webster * \\ Institute of Non-Newtonian Fluid Mechanics \\ University of Wales, Swansea SA2 8PP \\ email: m.f.webster@swansea.ac.uk
}

\begin{abstract}
A time-stepping finite element method is used to predict the viscoelastic stresses that arise in a tube-tooling wire-coating problem. The polymer melt HDPE is modelled by a multi-mode Phan-Thien/Tanner constitutive equation. Different flow geometries are considered to address optimisation of the process with respect to minimising the stress induced within the coating produced. The influence of the die itself and the various modes are considered. Relaxation times range for a three-mode model from $10^{-2}$ to $10^{2} s$ and for a seven-mode model from $10^{-3}$ to $10^{3} s$. Typical Weissenberg numbers may range up to $10^{4}$. Three modes are sufficient to adequately describe the flow, and shorter/narrower draw-down regions are identified as being preferable. Once an adequate land length has been gathered, that has relaxed the flow stresses prior to draw-down, the actual details of die design are found to be inconsequential to the induced stresses in the delivered coatings.

KEY WORDS: Multimode, viscoelastic, polymer melts, wire-coating, simulation.
\end{abstract}

\section{Introduction}

This paper addresses the issue of optimal process design for industrial wire-coating. We concentrate attention on a single tooling type, that of tube-tooling used primarily for widebore cable coating, for a high-density Polyethylene (HDPE) grade polymer. In this version of tooling design, the hot polymer melt is driven under pressure through an annular die, before being drawn down in a converging section to meet the fast moving wire. The die itself is composed of three sections, a straight inlet tube, a converging cone section, and a land region. The land region is the last section of the die just prior to die exit, whose purpose is to relax out stress from the flow before entering the draw-down region. Process conditions are such that the problem is amenable to an isothermal [1, 2], incompressible analysis [3] for steady-state flow. Levels of inertia are low, with typical Reynolds numbers of $\mathrm{O}\left(10^{-4}\right)$ and the flow is axisymmetric. No slip conditions are assumed within the die and on the wire, whilst free surfaces apply beyond the die. The location of the free surface for tube-tooling is fairly well categorised from practical experience for such an extension-dominated draw-down flow. This provides for a prescribed free surface and fixed melt-cable contact point.

The fundamental question under investigation in this study is how to optimise the process in hand, with respect to the minimisation of the stress imparted to the coating on the wire itself. This locked-in residual stress influences product quality giving rise to effects such as coating shrink-back. We note that increased stressing in the draw-down is undesirable, as the coating on the wire may display air pockets once the polymer experiences shrink-back.

${ }^{*}$ Author for correspondence 
In the investigation, a number of variations of the process geometry are considered using a viscoelastic analysis, that is performed here via a multi-mode implementation.

There are several distinct facets to this study. We consider a number of different die designs to ascertain gross flow effects and gain insight into the complex dynamics of the flow, that embodies a combination of shear and extensional flows in different proportions at various locations. We consider separately radial and axial adjustments, paying particular close attention to comparisons of stress profiles in the coating region. We also investigate individual mode contributions for various instances and effects of variation in boundary condition on entry to the land region. The latter issue is specifically directed towards the determination of die design influence on the process. We contrast the dependency of results upon the constitutive model, where we consider increasing the number of relaxation modes employed to represent the rheometrical properties of the melt from three to seven. For pragmatic reasons, we seek to minimise the number of modes to adequately represent the material response in this particular flow scenario.

The modelling of wire-coating extrusion has had extensive coverage in the literature $[4$, $1,2,5,3,6,7]$, though work has been devoted principally to pressure-tooling for narrow gauge wires. In contrast, tube-tooling (for which melt-wire contact is beyond the die) has received comparatively little attention. Molten polymers exhibit elastic behaviour under large deformation [8], yet till relatively recently, numerical solution techniques were incapable of reaching the high levels of elasticity $[5,9]$ involved to provide solutions to these flows. The literature abounds with earlier attempts that address this shortcoming, incorporating either an inelastic or lubrication approximation (see e.g. [4, 5, 6, 10, 11]). Nevertheless, in Binding et al. [12], it became apparent for example, that an inelastic view would not suffice to predict the appropriate levels of pressure-drop involved. Neither could stress be adequately modelled after this fashion, hence necessitating viscoelastic modelling. Our initial work on viscoelasticity lead to findings for an exponential single-mode Phan-Thien/Tanner (PTT) [13] model and established the technology to deal with such flows, where the high deformation rates imply short time-scales of operation and high levels of elasticity number (Weissenberg numbers of $\left.\mathrm{O}\left(10^{2}, 10^{4}\right)\right)[14,15,16]$. This is a stringent requirement, calling upon state-ofthe-art numerical techniques [17].

The present study is conducted under a viscoelastic treatment that also incorporates multi-mode representation, see also $[18,19,20]$. Earlier multi-mode work was compared with single-mode analyses [21], where we sought to extol the virtues and shortcomings of either approach. The present paper advances this theme, and in particular focuses on the more immediate issues relating to process optimisation, which is at the heart of the industrial problem. The particular numerical scheme adopted is a finite element based algorithm, based on a combination of Taylor-Galerkin and pressure-correction methods. This produces a semiimplicit time-stepping algorithm. Additional considerations for the discrete approximation of the differential constitutive equation require a consistent streamline upwinding method (SUPG) [22] and a Recovery method for velocity gradients [23, 24]. The latter ensures the accurate and continuous representation of secondary variable fields. A continuation approach, described below, is employed for these multi-mode model problems.

\section{Basic equations and numerical scheme}

We first introduce the basic equations that govern the problem. The equation of motion and continuity for an incompressible fluid of density $\rho$ are given by

$$
\rho \frac{\partial \boldsymbol{u}}{\partial t}=\nabla \cdot \boldsymbol{T}-\rho \boldsymbol{u} \cdot \nabla \boldsymbol{u}-\nabla p
$$


and

$$
\nabla \cdot \boldsymbol{u}=0
$$

where $u$ is the velocity vector, and $p$ the pressure. We chose to approximate the rheology of the coating polymer using an exponential Phan-Thien/Tanner model with multiple relaxation modes. The extra-stress tensor for the PTT model $[13,25]$ for modes $(i)$ is defined by

$$
\boldsymbol{T}=\sum_{i} \boldsymbol{\tau}_{i}+2 \mu_{2} \boldsymbol{D}
$$

so that for each mode $(i)$

$$
\begin{gathered}
f \boldsymbol{\tau}_{i}+\lambda_{1}^{i} \stackrel{\square}{\boldsymbol{\tau}}_{i}=2 \mu_{1}^{i} \boldsymbol{D}, \\
\stackrel{\square}{\boldsymbol{\tau}}_{i}=\left(1-\frac{\xi}{2}\right) \stackrel{\nabla}{\boldsymbol{\tau}}_{i}+\frac{\xi}{2} \overrightarrow{\boldsymbol{\tau}}_{i}=\stackrel{\nabla}{\boldsymbol{\tau}}_{i}+\xi\left(\boldsymbol{D} \cdot \boldsymbol{\tau}_{i}+\boldsymbol{\tau}_{i} \cdot \boldsymbol{D}\right), \\
f=\exp \left[\frac{\epsilon \lambda_{1}^{i}}{\mu_{1}^{i}} \operatorname{trace}\left(\boldsymbol{\tau}_{i}\right)\right] .
\end{gathered}
$$

Here, $\boldsymbol{D}$ is the rate of deformation tensor,

$$
\boldsymbol{D}=\frac{1}{2}\left(\nabla \boldsymbol{u}+\nabla \boldsymbol{u}^{\dagger}\right)=\frac{1}{2}\left(L+L^{\dagger}\right)
$$

$\lambda_{1}^{i}, \mu_{1}^{i}$ are the relaxation time and a polymeric viscosity for mode $(i)$, and $\mu_{2}$ is a solvent viscosity. According to [26] and [27] for such polymer melts, the solvent viscosity is negligible compared to the polymeric part, being less than $\mathrm{O}\left(0.001 \mu_{0}^{i}\right)$, and hence may be ignored. The total zero-shear viscosity is defined viz

$$
\mu_{0}=\sum_{i} \mu_{0}^{i}=\mu_{2}+\sum_{i} \mu_{1}^{i}=\sum_{i} \mu_{1}^{i}
$$

The model constants $\epsilon$ and $\xi,(\epsilon \geq 0,0 \leq \xi \leq 2)$ are non-dimensional parameters that must be determined by fitting to the experimental data for shear and/or elongational viscosities. The set $(\epsilon=1.0, \xi=0)$ is found to be an appropriate choice to approximate the HDPE grade polymer considered here.

Non-dimensionalisation is introduced through scales of $\mathrm{L}$ and $\mathrm{U}$ for length and velocity respectively, as follows

$$
x^{\star}=\frac{x}{L} \text { and } \boldsymbol{u}^{\star}=\frac{u}{U} .
$$

Non-dimensional numbers of Reynolds and Weissenberg numbers, $R e$ and $W e$, are introduced, defined as

$$
R e=\frac{\rho U L}{\mu_{0}} \text { and } W e^{i}=\lambda_{1}^{i} \frac{U}{L},
$$

for which, $W e^{i}$ corresponds to mode $(i)$.

Multi-mode computations necessitate initially a single-mode solution to generate a first approximation to the base kinematics for the flow. These fields are then frozen and each individual stress mode is computed, before their combination is used to recompute a correction to the kinematics. In the multi-mode context, no solvent viscosity is incorporated. The target level of Weissenberg number is relatively high for such computations and this necessitates some sophisticated numerical treatment, following Ref.[17]. For illustration, let 
us consider the single-mode scenario for a Weissenberg number of 331, corresponding to the highest relaxation time of the three-mode model. Commencing from a converged Newtonian kinematic field and quiescent initial stress conditions, a coupled single-mode PTT solution is sought using a small solvent viscosity, of say $\mu_{2}=0.01 \mu_{0}$. Inlet stress boundary conditions are developed themselves in a temporal manner, as part of the solution process. This provides a first guess for the kinematics of the multi-mode problem, to commence the procedure outlined below. The success of this scheme in attaining such large Weissenberg number solutions is due to several factors: the enhanced stability properties of the transient SUPG recovery implementation itself; the evolution of consistent temporal boundary conditions from appropriate initial conditions; the continuation procedure adopted for seeking solutions at certain sensitive levels of material parameters, such as $\mu_{1}$; adopting $\xi=0$ avoids some start-up shear instabilities.

To solve equations 1-6, we employ a four-staged process. The first stage is to calculate a Newtonian field $(\boldsymbol{u}, p)$, as in [28]. At a second stage a single-mode viscoelastic solution is computed, starting from the Newtonian solution and quiescent initial stress conditions. At a third stage and commencing from such a single-mode solution, each of the three stress modes is calculated in a decoupled sense, on resetting the solvent viscosity to zero and freezing the viscoelastic velocity field. For each mode $(i)$, the viscosity, pressure and stress are nondimensionalised as follows

$$
\left(\mu^{i}\right)^{\star}=\frac{\mu^{i}}{\mu_{0}^{i}}, p^{\star}=\mu_{0}^{i} \frac{U}{L} p \text { and } \tau_{i}^{\star}=\mu_{0}^{i} \frac{U}{L} \tau_{i}
$$

The total stress is then gathered through Eq. 3, at a fourth and final stage. Updated velocity and pressure fields are recomputed by freezing the total stress. Here nondimensionalisation is performed according to the total zero-shear viscosity $\mu_{0}$. This procedure is noted to correct the pressure field. Meanwhile variation in the velocity field is found to be insignificantly small, rendering reevaluation unnecessary.

Time steps of $\mathrm{O}\left(10^{-3}\right)$ are used and convergence to a steady state is monitored via a relative temporal increment $l_{2}$ norm on the solution taken to a tolerance of $\mathrm{O}\left(10^{-6}\right)$, see [17] for detail further.

At stage 2, a coupled Recovery-based scheme [17] is employed with a solvent viscosity of $\mu_{2}=0.01 \mu_{0}$ to derive the viscoelastic kinematics. This is accomplished through a second order Taylor-Galerkin/pressure correction scheme. Two steps are employed for the stress equations (see Townsend and Webster [29]). A breakdown into fractional stages may be introduced via semi-discretisation in time to demonstrate the appropriate scheme structure. Step 1a :

$$
\begin{aligned}
& \frac{2 R e}{\triangle t}\left(\boldsymbol{u}^{n+\frac{1}{2}}-\boldsymbol{u}^{n}\right)=\left[\nabla \cdot\left(2 \mu_{2} \boldsymbol{D}+\boldsymbol{\tau}\right)-\operatorname{Re} \boldsymbol{u} \cdot \nabla \boldsymbol{u}-\nabla p\right]^{n} \\
& +\nabla \cdot \mu_{2}\left(\boldsymbol{D}^{n+\frac{1}{2}}-\boldsymbol{D}^{n}\right), \\
& \frac{2 W e}{\triangle t}\left(\boldsymbol{\tau}^{n+\frac{1}{2}}-\boldsymbol{\tau}^{n}\right)=\left[2 \mu_{1} \boldsymbol{D}-f \boldsymbol{\tau}-W e\left\{\boldsymbol{u} \cdot \nabla \boldsymbol{\tau}-\boldsymbol{\tau} \cdot \nabla \boldsymbol{u}-(\boldsymbol{\tau} \cdot \nabla \boldsymbol{u})^{\dagger}\right\}\right]^{n},
\end{aligned}
$$

Step 1b :

$$
\begin{gathered}
\frac{R e}{\triangle t}\left(\boldsymbol{u}^{\star}-\boldsymbol{u}^{n}\right)=[\nabla \cdot \boldsymbol{\tau}-R e \boldsymbol{u} \cdot \nabla \boldsymbol{u}]^{n+\frac{1}{2}} \\
+\left[\nabla \cdot\left(2 \mu_{1} \boldsymbol{D}\right)-\nabla p\right]^{n} \\
+\nabla \cdot \mu_{2}\left(\boldsymbol{D}^{\star}-\boldsymbol{D}^{n}\right), \\
\frac{W e}{\triangle t}\left(\boldsymbol{\tau}^{n+1}-\boldsymbol{\tau}^{n+\frac{1}{2}}\right)=\left[2 \mu_{1} \boldsymbol{D}-f \boldsymbol{\tau}-W e\left\{\boldsymbol{u} \cdot \nabla \boldsymbol{\tau}-\boldsymbol{\tau} \cdot \nabla \boldsymbol{u}-(\boldsymbol{\tau} \cdot \nabla \boldsymbol{u})^{\dagger}\right\}\right]^{n+\frac{1}{2}},
\end{gathered}
$$


where $\boldsymbol{u}^{\star}$ is an intermediate velocity field that is non-divergence free. The pressure is evaluated through a temporal increment equation

Step2:

$$
\nabla^{2}\left(p^{n+1}-p^{n}\right)=\frac{2 R e}{\triangle t} \nabla \cdot u^{\star}
$$

and the end-of-time step velocity is computed via the correction equation Step 3:

$$
\frac{2 R e}{\triangle t}\left(\boldsymbol{u}^{n+1}-\boldsymbol{u}^{\star}\right)=-\nabla\left(p^{n+1}-p^{n}\right)
$$

Note, the predictor-corrector structure of the first step, that embodies both implicit and explicit parts, and the pressure-correction form in the second and third steps, where temporal solution differences are computed. The third step computes a correction to the velocity field, enforcing incompressibility on the time step to the order of the scheme. Diffusion terms are approximated with a Crank Nicolson discretisation over a time step, which introduces implicitness and stability to the procedure.

The finite element discretisation employs piecewise-continuous quadratic interpolation on triangles for velocity and stress, and linear interpolation for pressure. Spatial weighting is generally of a Galerkin form, with the exception of the constitutive equation, where consistent streamline upwinding is used. This and recovery-based methods are stability enhancing techniques of particular significance at high levels of elasticity, as involved here. A direct method of solution is employed to solve for the pressure equation step, whilst indirect Jacobi iteration is invoked for the remaining steps.

Let us take

$$
\boldsymbol{u}, \boldsymbol{\tau}=\sum_{j=1}^{N_{v}} \phi_{j}\left(\boldsymbol{U}_{j}, \boldsymbol{T}_{j}\right), \text { and } p=\sum_{j=1}^{N_{p}} \psi_{j} P_{j},
$$

where $N_{v}$ and $N_{p}$ represent the number of quadratic and linear interpolation points per element, and $\phi_{i}, \psi_{i}$ are quadratic and linear shape functions, respectively. A Galerkin approach is implemented for the velocity and pressure. In contrast, For the constitutive equations, the SUPG method is used, and the weighting function $\phi_{i}$ is modified to $\left(1+\alpha_{h} \boldsymbol{u} \cdot \nabla\right) \phi_{i}$, where $\alpha_{h}$ is defined as in Ref. [30]. In addition, by recovery, we have

$$
\boldsymbol{L}=\sum_{j=1}^{N_{v}} \phi_{j} \boldsymbol{L}_{j}
$$

We find it convenient to refer to components of $\boldsymbol{T}$ through linearisation as $\left(\boldsymbol{T}_{r r}, \boldsymbol{T}_{r z}, \boldsymbol{T}_{z z}, \boldsymbol{T}_{\theta \theta}\right)$. In matrix form, Eqs. 12-17 are translated in the following manner.

Step 1a :

$$
\begin{gathered}
\left(\frac{2 R e}{\triangle t} M+\frac{\mu_{2}}{2} S\right)\left(\boldsymbol{U}^{n+\frac{1}{2}}-\boldsymbol{U}^{n}\right)=\left\{-\left[\mu_{2} S+\operatorname{ReN}(\boldsymbol{U})\right] \boldsymbol{U}-B \boldsymbol{T}\right\}^{n}+L^{T} \boldsymbol{P}^{n} \\
\frac{2 D e}{\triangle t} M\left(\boldsymbol{T}^{n+\frac{1}{2}}-\boldsymbol{T}^{n}\right)=\left[2 \mu_{1} M\left(\boldsymbol{L}+\boldsymbol{L}^{\dagger}\right)-\{M+W e N(\boldsymbol{U})\} \boldsymbol{T}+W e\left\{N_{1}(\boldsymbol{T}) \boldsymbol{L}+\left(N_{1}(\boldsymbol{T}) \boldsymbol{L}\right)^{\dagger}\right\}\right]^{n},
\end{gathered}
$$

Step 1b :

$$
\begin{gathered}
\left(\frac{R e}{\triangle t} M+\frac{\mu_{2}}{2} S\right)\left(\boldsymbol{U}^{\star}-\boldsymbol{U}^{n}\right)=\left\{-\left[\mu_{2} S+R e N(\boldsymbol{U})\right] \boldsymbol{U}-B \boldsymbol{T}\right\}^{n+\frac{1}{2}}+L^{T} \boldsymbol{P}^{n} \\
\frac{D e}{\triangle t} M\left(\boldsymbol{T}^{n+1}-\boldsymbol{T}^{n}\right)=\left[2 \mu_{1} M\left(\boldsymbol{L}+\boldsymbol{L}^{\dagger}\right)-\{M+W e N(\boldsymbol{U})\} \boldsymbol{T}+W e\left\{N_{1}(\boldsymbol{T}) \boldsymbol{L}+\left(N_{1}(\boldsymbol{T}) \boldsymbol{L}\right)^{\dagger}\right\}\right]^{n+\frac{1}{2}}
\end{gathered}
$$


Step 2 :

$$
-K\left(\boldsymbol{P}^{n+1}-\boldsymbol{P}^{n}\right)=\frac{2 R e}{\Delta t} L \boldsymbol{U}^{\star}
$$

Step 3 :

$$
\frac{2 R e}{\triangle t} M\left(\boldsymbol{U}^{n+1}-\boldsymbol{U}^{\star}\right)=-R^{T}\left(\boldsymbol{P}^{n+1}-\boldsymbol{P}^{n}\right),
$$

where $M, S$ and $N$ are the consistent mass matrix, the diffusive matrix and the advection matrix, respectively, defined as

$$
\begin{aligned}
& (M)_{i j}=\int_{\Omega} \phi_{i} \phi_{j} d \Omega, S=\left[\begin{array}{ll}
S_{11} & S_{12} \\
S_{12}^{\dagger} & S_{22}
\end{array}\right], \\
& \left(S_{11}\right)_{i j}=\int_{\Omega}\left\{2 \frac{\partial \phi_{i}}{\partial r} \frac{\partial \phi_{j}}{\partial r}+\frac{\partial \phi_{i}}{\partial z} \frac{\partial \phi_{j}}{\partial z}+2 \frac{\phi_{i} \phi_{j}}{r^{2}}\right\} d \Omega, \\
& \left(S_{12}\right)_{i j}=\int_{\Omega} \frac{\partial \phi_{i}}{\partial z} \frac{\partial \phi_{j}}{\partial r} d \Omega \\
& \left(S_{22}\right)_{i j}=\int_{\Omega}\left\{\frac{\partial \phi_{i}}{\partial r} \frac{\partial \phi_{j}}{\partial r}+2 \frac{\partial \phi_{i}}{\partial z} \frac{\partial \phi_{j}}{\partial z}\right\} d \Omega, \\
& (M)_{i j}=\int_{\Omega} \phi_{i} \phi_{j} d \Omega,(N(\boldsymbol{U}))_{i j}=\int_{\Omega} \phi_{i} \phi_{l} \boldsymbol{U}_{l} \nabla \phi_{j} d \Omega, \\
& \left(N_{1}(\boldsymbol{T})\right)_{i j}=\int_{\Omega} \phi_{i} \phi_{l} \boldsymbol{T}_{l} \phi_{j} d \Omega, d \Omega=r d r d z . \\
& B=\left[\begin{array}{cccc}
B_{1}-B_{3} & B_{2} & 0 & B_{3} \\
0 & B_{1} & B_{2} & 0
\end{array}\right], \\
& \left(B_{1}\right)_{i j}=\int_{\Omega} \phi_{i} \frac{\partial \phi_{j}}{\partial r} d \Omega,\left(B_{2}\right)_{i j}=\int_{\Omega} \phi_{i} \frac{\partial \phi_{j}}{\partial z} d \Omega,\left(B_{3}\right)_{i j}=\int_{\Omega} \frac{\phi_{i} \phi_{j}}{r} d \Omega,
\end{aligned}
$$

The pressure stiffness matrix $K$, and vector $F_{1}$, and the remaining matrices are given viz

$$
\begin{gathered}
(K)_{i j}=\int_{\Omega} \nabla \psi_{i} \nabla \psi_{j} d \Omega, \\
L=\left(L_{1}, L_{2}\right),\left(L_{k}\right)_{i j}=\int_{\Omega} \psi_{i} \frac{\partial \phi_{j}}{\partial x_{k}} d \Omega, \\
\left(R_{k}\right)_{i j}=\int_{\Omega} \frac{\partial \psi_{i}}{\partial x_{k}} \phi_{j} d \Omega .
\end{gathered}
$$

As cited in Townsend and Webster [29], and due to the freedom of choice of $\boldsymbol{u}^{\star}$, we can choose $\boldsymbol{u}^{\star}=\boldsymbol{u}^{n+1}$ on the domain boundary, which simplifies the treatment of boundary condition for $p^{n+1}-p^{n}$. Hence, homogeneous Neumann conditions are imposed on $\left(p^{n+1}-p^{n}\right)$, that arise naturally in the weak weighted-residual form for steady flows. This is observed in Eq. 23 at the boundary. For time-independent boundary conditions, this choice is exact. For timedependent boundary conditions, for smooth pressures $p^{n+1}-p^{n}=O(\Delta t)$; therefore by Eq. 17 , the error in the boundary conditions will be $O\left(\Delta t^{2}\right)$ [31]. A fixed datum on $\left(p^{n+1}-p^{n}\right)$ completes the specification. 


\section{Problem specification}

In this study we perform an extensive analysis of geometric adjustment. A schematic representation of each of the various geometries is provided in Figure 1, that covers Cases a) through $\mathrm{f}$ ). The relative dimensions are given in Table 1 . In earlier studies, the design of Case a) was employed to represent the combined tube-tooling/draw-down flow [15, 16, 21]. Radial adjustments alter draw-down ratios and inlet tube-bore. Widening inlet tube-bore at the same flowrate reduces the level of shearing imparted to this initial flow. Reduction in the draw-down ratio enhances stress levels. Axial modifications within the converging tube of the die and the draw-down section are used to categorise the dependency of flow behaviour upon each portion of the flow. In this manner, we are able to ascertain, criticality of the complex flow interactions present, and the effect of multiple modes on stress production in the coating.

In Case b), the draw-down section is halved in length, whilst the remaining parameters are held fixed. Adjustment of the converging cone is studied by halving the distance $L_{2}$, as in Case c). Both Cases b) and c) are combined in Case d). In contrast for Case e), the bore is reduced of the land region, the last die section. Case f) is derived from Case e) by widening the inlet tube.

Boundary conditions are taken as no-slip within the annular die with a prescribed inlet pressure-driven flow at a given flowrate. Externally to the die, no-slip applies on the wire, and the free surfaces are taken at locations provided from experimental evidence. Velocity boundary conditions on the free surfaces are then approximated assuming localised plug flow throughout the draw-down and coating regions. A typical processing temperature is $200^{\circ} C$. The rheological data in shear, $\mu_{s}$, and extensional viscosity, $\mu_{e}$, as displayed in Figure 3, Table 2 and 3 (see on for discussion), are detailed in [27], from which the relevant material parameters are calculated. The shear data is taken from steady shear experiments, whilst the extensional data is derived from an approximate energy balance argument based on contraction flows [32]. For the actual process, the shear rates range over values up to $\mathrm{O}\left(10^{3} s^{-1}\right)$, whilst extension rates reach values of $\mathrm{O}\left(10^{2} s^{-1}\right)$.

The characteristic velocity is that of the wire, $u=0.334 \mathrm{~m} / \mathrm{s}$, and the characteristic length is taken as the standard length of the draw-down section, $L$, equating to $L_{4}$ of Case a). The geometry of Case a) is used when considering comparison between the settings of different relaxation times.

Various meshes have been employed in this work to assess accuracy. We have checked our solutions for mesh convergence elsewhere, based on Case a) and draw-down flow. This we have found to be satisfactory. A representative finite element mesh composed of triangles is displayed in Figure 2, taking the die and draw-down sections separately to aid viewing of the structure. A summary of mesh characteristics on the different sections of the domain is given in Table 4 and Table 5, where both total numbers of elements and regional distributions are included.

\section{Geometry adjustment analysis}

An analysis of the influence of geometric changes on the stress distribution is presented in the form of streamwise stress profiles, in tensile $\left(\tau_{z z}\right)$ and shear $\left(\tau_{r z}\right)$ components. Axial and radial adjustments to the process design configuration are considered separately. 


\subsection{Axial variations: Cases a) - d)}

We may take results in comparative terms by reference to Case a). Figure 4 demonstrates the general trends in streamwise stress response throughout the flow, where in the first instance the sampling line chosen is along the inner radius of the die and extrudate. These plots reflect the influence of the boundaries, and in particular, the localised effects of the die-exit point discontinuity, recorded at axial location $\mathrm{z}=0$ as indicated. We subsequently proceed to contrast this view against core flow plots, where such boundary and singularity influences are minimised. In absolute terms, levels of stress rise within the converging cone from that for an annular fully-developed inlet flow, undergo some rearrangement across the land length, decay sharply at die-exit, and then gradually relax in the draw-down and on the wire. There are rapid changes at entry to the converging cone and land region, though noteably, the melt-cable contact point is traversed relatively smoothly. This justifies the modelling approximation of adopting a fixed melt-cable contact point.

Specifically for Case a), the tensile stress has dropped in maximum value from $0.2 \mathrm{MPa}$ at die-entry to $0.08 \mathrm{MPa}$ in the exit coating. Extrema occur at entry and exit of the land region, reaching a value approaching 0.6 $\mathrm{MPa}$ at die-exit. Once the draw-down has been encountered, there is only minor stress relaxation evident. Comparably, the shear stress is 60 percent of the tensile stress in magnitude, and diminishes by about one third from entry to exit.

Case b) is associated with halving the draw-down length, $L_{4}$, as illustrated in Figure $1 \mathrm{~b}$. The major flow adjustments occur in the draw-down and on the wire as to be expected. This may be observed in contrasting Case a) against Case b) on the stress profiles of Figure 4. The extrema of stress in the draw-down are larger than those of Case a), tripling for $\tau_{r z}$ to around $0.15 \mathrm{MPa}$ and for $\tau_{z z}$ to $0.3 \mathrm{MPa}$.

In all instances, both tensile and shear stress drop sharply at die exit, but less so in Case b) than Case a). Subsequently, the slight rise, decay and upturn of $\tau_{z z}$ prior to meeting the wire of Case a), now assumes the form of a more gradual bowing out for Case b). On the wire for Case b), the tensile stress starts to decay from a higher peak value than in Case a), and the decay rate is greater. This leads to a diminished maximum exit stress level, varying from 0.08 MPa for Case a) to 0.06 MPa for Case b). Most noteably, Case b) has not reached a fully-developed plug flow at the domain exit, indicating that there is still further scope for stress relaxation in the coating flow.

Along the wire, the decrease is 25 percent greater than for Case a) in normal stress, and 50 percent in shear stress. These changes may be explained through the increase in elongation rate, $\left(\dot{\epsilon}=\frac{u}{L}\right.$, when $L$ is decreased, then $\dot{\epsilon}$ increases), and the reduced influence of shear stress. As the polymer is extension thinning, the elongational viscosity consequently decreases and the stress modifies accordingly.

Case c) involves maintaining the draw-down section as in the standard Case a), and halving the converging cone length, $L_{2}$, as illustrated in Figure 1c. This generates larger shearing in the converging cone. Convergence of the simulation process becomes increasingly more difficult, requiring greater mesh refinement in the converging cone and land region. A uniform refinement is employed. The meshing details are provided in Table 4 and illustrated in Figure 2. The shear stress, as observed in the streamwise profile plot of Figure 4, attains a peak absolute value of $0.3 \mathrm{MPa}$ towards the termination of the converging cone, i.e. tripling the value corresponding to Case a). In contrast, there are no significant adjustments in flow behaviour observed within the land, draw-down and wire regions, so that approximately Case a) values are reproduced. There is simply a more rapid build-up to the stress levels of the land region over a shorter converging cone of sharper angle. We can conclude that decreasing 
the length of the converging cone in this manner is inadvisable, as it only serves to increase the shear rate without any tangible benefits.

Both Cases b) and c) are combined to form Case d); Figure 1d) illustrates the relevant dimensions. The decrease in length of the converging cone generates the same flow response as in Case c), with an increase in shear rate and stress accordingly over Case a). Meanwhile, the reduction in length of the draw-down section replicates the results of Case b), with a sharp decrease of the stress, as noted above. Hence, it is deemed unnecessary to reproduce plots for this case.

\subsection{Radial variations: Cases a), e) and f)}

In Case e), narrowing the bore of the land region, by decreasing radial dimensions $\left(R_{3}\right.$, $R_{4}$ ), affects the angle of the draw-down cone and reduces its slope. The draw-down ratio is decreased from 2.0 for Case a) to 1.44 for Case e). A schematic diagram for this instance is shown in Figure 1e).

By comparing the curves of Figure 5 for Cases a) and e) within the die, only a slight increase in shear stress is observed in the converging cone and land region. In tensile stress, there is more noticeable increase on the land region. In contrast, the value of the tensile stress doubles from Case a) to e) just beyond the start of the draw-down cone, before encountering a sharp decrease. Here, the shear stress level is also amplified compared with Case a). This consequently influences the pattern of behaviour for the normal stress. Along the wire, the stress decreases and is lower in value than that of Case a). For Case e), plug flow has not been fully realised, whilst the converse is true in Case a).

For the final case studied here, that of Case $\mathrm{f}$ ), the inlet tube is widened over that for Case e). According to Figures 5, this design adjustment has its largest impact upon the flow in the tube-tooling die sections, particularly over the inlet and converging cone. As the tube is wider and the same flow rate is maintained, this leads to a reduction (halving) in stress levels. Nevertheless there is greater build-up in stress within the converging cone, to eventually reach an equivalent peak level in the land region as encountered for Case e). Hence, this adjustment has no impact on the stress imparted to the coating.

\subsection{Core flow results: Cases a) - f)}

Figures 6 and 7 are included to isolate core flow stress behaviour and are to be taken in contrast to the results depicted in Figure 4, Cases a) to c), and Figure 5, Cases e) to f). The principal feature here is the sharp rise encountered at the entry to the land region and the absence of such at the die-exit location, where the flow enters the draw-down section. These findings reveal essentially a lack of dependency of the stress in the final coating to the influence of the die-exit singularity. Core flow levels of shear stress are minimal in the coating. The lowest levels of tensile stress are provided by reduced draw-down alternatives in Cases e) and f). Cases b) and d), with shortened draw-down lengths also would indicate a similar trend but require a comparable draw-down/wire section length to equate to the domain exit stress levels of Cases e) and f). Cross-stream variations are noted between core and inner wall plots, that confirm the need for a two-dimensional treatment.

\subsection{Comparison for wire coating region}

Streamwise profiles for Cases a), b) and e) are provided in Figure 8. Only the results on the inner coating surface are plotted as these suffice to demonstrate the overall trends. Cases b) and e) convey the minimal coating stress levels at around $40 \mathrm{KPa}$ in tensile stress at domain 
exit. This is about half the value observed for Case a). Shear stress is then about half of the tensile stress in each instant.

Cross-stream variations in tensile stress reveal a central minimum peak prominent for Cases e) and $\mathrm{f}$ ). This confirms that plug flow has not been fully achieved at domain exit in these instances. The same is true for Cases b) and d), but to a lesser extent.

\subsection{Individual mode contributions: Case a), b) and e)}

In order to provide some insight into the stress response upon shortening the draw-down (as in Case b) of Figure 4), we analyse the various contributions from each mode in Figure 9. The conclusion is that the exaggerated decay in the shortened draw-down region is related to the shortest mode response and the halving of residence time over this region, to around 0.18 seconds. The determining factor is the relative significance of the shortest mode $(0.017 \mathrm{~s})$ to the second shortest mode $(0.87 \mathrm{~s})$ for the residence time considered.

Similarly, in Figure 10 we analyse the influence of draw-down ratio reduction in comparison to Figure 5. Here, we see again that reduction of residence time amplifies the dominance of the shortest mode, generating greater stress relaxation in the draw-down. A comparable plot for Case a) and individual modes is presented in Figure 11, where one can observe the significant variations generated in draw-down for the shortest mode.

\subsection{Land region boundary condition variation: Case a)}

Figure 12 displays the effect of adjusting the inlet flow to the land region for Case a) and the more dominant tensile stress. Streamwise profiles are taken on sample lines along the inner coating surface and in core flow. There are two instances cited, that for Case a) as above with entry conditions emanating from a converging cone, and that for a fully-developed shear flow. The results reveal that the land length has performed its role of stress relaxation. Hence, in this scenario and in particular with the chosen land length specification, there is little influence of the die design on the coating stress. The significance of this finding to the processing is large, as it implies that it does not matter greatly what design of die is employed provided the stress levels delivered to the draw-down are minimal. This is consistent with the results derived for Cases c) and f).

\section{Three to seven-mode comparison}

The HDPE polymer is modelled using both three and seven relaxation times. Earlier work compared a range of polymeric materials including LDPE and HDPE [21], and also LDPE alone $[15,16]$. HDPE has a linear structure molecular topology, as opposed to LDPE that possesses a branched structure. This difference is responsible for providing disparate bulk rheological behaviour. The various material parameters involved are provided in Tables 2 and 3 (see [26] and [27]). From previous studies, the residence time across the flow station was calculated as $t_{r e s}=2.5 s$. Accordingly, from Table 3 , for modes with relaxation times $\lambda_{i}$ greater than $18 \mathrm{~s}$, the fluid will not have sufficient time to react and stress behaviour will be essentially constant throughout the geometry.

The shear and elongational viscosities, plotted in Figure 3, indicate that both three and seven-mode models adopt similar overall material response, with both models displaying comparable magnitudes over shear/elongational rate ranges of $\left[10^{-1}, 10^{2} s^{-1}\right]$. There are only minor differences between the fits and the data outside this range. For the elongational viscosity, both models reflect a uniform shift from the experimental data. A shift towards 
the experimental $\mu_{e}$ values may be achieved by incorporating a solvent viscosity component $\mathrm{O}\left(10^{-2} \mu_{0}\right)$; however this factor will also elevate $\mu_{s}$ away from the experimental $\mu_{s}$ data that is anticipated to be reliable. Note, the experimental data is actually recorded for $\mu_{e}$ over $[10$, $\left.10^{3} \mathrm{~s}^{-1}\right]$, and for shear $\left[10^{-2}, 10^{4} \mathrm{~s}^{-1}\right]$ as in [27].

In Figure 13, the shear and normal stresses are plotted for comparison between three and seven-mode models along the inner tube wall/draw down side in the streamwise direction. It is clear that, there are no major differences to observe in the flow, except some increase of the stress magnitude in the converging tube and land region. Of most significance is that stress levels in the coating on the wire are practically indistinguishable. Hence the three-mode model is demonstrated to be an adequate choice. The increase of the stress magnitude in the converging tube and land region is due to the adjustments in the fit employed for the shear viscosity, as depicted in Figure 3a. At high shear rates, for both three and seven-modes, the fit in Figure 3a shows that the shear viscosity for seven-modes is slightly higher than that of three-modes, resulting in higher normal stress values. The values of both shear and normal stress at the end of the converging tube and in the land region depend on the higher shear rates encountered there, being typically of $\mathrm{O}\left(10^{3} s^{-1}\right)$.

Figure 14 is included to provide some insight into the relative significance of the individual modes of the seven-mode instance. It is apparent that those modes corresponding to time scales above $18 \mathrm{~s}$ have little presence in the total stress, remaining lower than $0.03 \mathrm{MPa}$ in attenuation. The relative response of the third mode $\left(\lambda_{1}=0.29 \mathrm{~s}\right)$ and fourth mode $\left(\lambda_{1}=2.27 s\right)$ is segregated, and these carry the greatest contribution to the stress in the coating. The discrete relaxation spectrum of the three-mode model achieves a similar stress response, where the dominant contributions are associated with the first mode $\left(\lambda_{1}=0.017 \mathrm{~s}\right)$ and the second mode $\left(\lambda_{1}=0.87 \mathrm{~s}\right)$. These findings are consistent with the observation that the critical factor controlling the stress decay is the draw-down residence time (less than $0.5 \mathrm{~s}$ ) and its magnitude in relationship to the components of the discrete relaxation spectrum employed.

\section{Conclusions}

With respect to process optimisation and minimisation of flow-induced residual stressing in the coating, the most preferable scenario would appear to be a combination of Cases b) and e). These cases convey the minimal coating stress levels at around $40 \mathrm{KPa}$ in tensile stress and $20 \mathrm{KPa}$ in shear stress at domain exit. This is about half the value observed for Case a). There is also further scope for relaxation in options Case b), d), e) and f), as fully developed plug flow has not been reached along the wire at domain exit. Adjusting the die design, via decreasing the length of the converging cone or widening the inlet tube, serves no tangible benefits with little influence on the coating stress. Furthermore, this is confirmed by adjusting the inlet flow to the land region for Case a) and a fixed land length specification. The results reveal that the land length has performed its role of stress relaxation adequately.

From the various contributions from each of the three modes, the exaggerated decay in the shortened draw-down region may be attributed to the shortest mode response and the halving of residence time over this region, to around 0.18 seconds. The determining factor is found to be the relative significance of the shortest mode $(0.017 \mathrm{~s})$ to the second shortest mode $(0.87 \mathrm{~s})$ for the residence time considered. Similar arguments apply for reduction in the draw-down ratio, where we observe again that reduction of residence time amplifies the dominance of the shortest mode, generating greater stress relaxation in the draw-down.

There are only minor differences in stress response observed for the tube-tooling flow, between the use of three and seven-mode models. Hence, from an efficiency standpoint, it is 
preferable to use the three-mode model representation. Modes above 18s have little presence in the total stress. The critical factor controlling the stress decay is the draw-down residence time (less than $0.5 \mathrm{~s}$ ), that stimulates the dominance of specific modes within the discrete relaxation spectrum employed.

\section{Acknowledgements}

The authors wish to gratefully acknowledge the financial support and collaboration of BICC Cables Ltd. Thanks is also due to our colleagues, Professor Ken Walters FRS, Professor Russell Davies and Dr David Binding, within the Institute of non-Newtonian Fluid Mechanics for their support work on rheological characterisation. 


\section{References}

[1] G. Barakos and E. Mitsoulis. Non-isothermal viscoelastic simulations of extrusion through dies and prediction of the bending phenomenon. J. Non-Newtonian Fluid Mech., 62:55-79, 1996.

[2] J.F Carley, T. Endo, and W.B. Krantz. Realistic analysis of flow in wire-coating dies. Polymer Eng. Sci., 19 (16):1178-1187, 1979.

[3] T.J. Chung. Finite Element Analysis in Fluid Dynamics. McGraw-Hill International Book Company, USA, 1978.

[4] B. Arpin, P.G. Lafleur, and V. Lenir. Computer aided design of wire coating dies. ANTEC, 91:58-61, 1991.

[5] B. Caswell and R.I. Tanner. Wirecoating die design using finite element methods. Polymer Eng. Sci., 18 (5):416-421, 1978.

[6] C.D. Han and D. Rao. Studies on wire coating extrusion. I, The Rheology of Wire Coating Extrusion. Polymer Eng. Sci., 18 (13):1019-1029, 1978.

[7] R.T. Fenner and J.G. Williams. Trans. J. Plastics Inst., pages 701-706, 1967.

[8] Z. Tadmor and R.B. Bird. Rheological analysis of stabilizing forces in wire-coating dies. Polymer Eng. Sci., 14 (2):124-136, 1974.

[9] E. Mitsoulis, J. Vlachopoulos, and F.A. Mirza. Numerical simulation of entry and exit flows in slit dies. Polymer Eng. Sci., 24 (9):707-715, 1984.

[10] E. Mitsoulis. Fluid flow and heat transfer in wire coating: A review. Advances in Polymer Technology, 6 (4):467-487, 1986.

[11] E. Mitsoulis, R. Wagner, and F.L. Heng. Numerical simulation of wire-coating lowdensity polyethylene: Theory and experiments. Polymer Eng. Sci., 28 (5):291-311, 1988.

[12] D.M. Binding, A.R. Blythe, S. Gunter, A.A. Mosquera, P. Townsend, and M.F. Webster. Modelling polymer melt flows in wirecoating processes related fields. J. Non-Newtonian Fluid Mech., 64:191-206, 1996.

[13] N. Phan-Thien and R.I. Tanner. A new constitutive equation derived from network theory. J. Non-Newtonian Fluid Mech., 2:353-365, 1977.

[14] S. Gunter, P. Townsend, and M.F. Webster. Simulation of some model viscoelastic extensional flows. Int. J. Num. Meth. Fluids, 23:691-710, 1996.

[15] I. Mutlu, P. Townsend, and M.F. Webster. Simulation of cable-coating viscoelastic flows with coupled and decoupled schemes. J. Non-Newtonian Fluid Mech., 74:1-23, 1998.

[16] I. Mutlu, P. Townsend, and M.F. Webster. Computation of viscoelastic cable coating flows. Int. J. Numer. Meth. Fluids, 26:697-712, 1998.

[17] H. Matallah, P. Townsend, and M.F. Webster. Recovery and stress-splitting schemes for viscoelastic flows. J. Non-Newtonian Fluid Mech., 75:139-166, 1998. 
[18] F.P.T. Baaijens, S.H.A. Selen, H.P.W. Baaijens, G.W.M. Peters, and H.E.H. Meijer. Viscoelastic flow past a confined cylinder of a LDPE melt. J. Non-Newtonian Fluid Mech., 68:173-203, 1997.

[19] H.P.W. Baaijens. Evaluation of constitutive equations for polymer melts and solutions in complex flows. PhD. Thesis, Eindhoven University of Technology, Netherlands, 1994.

[20] C. Beraudo, A. Fortin, T. Coupez, Y. Demay, B. Vergnes, and J.F. Agassant. A finite element method for computing the flow of multi-mode viscoelastic fluids: comparison with experiments. J. Non-Newtonian Fluid Mech., 75:1-23, 1998.

[21] H. Matallah, P. Townsend, and M.F. Webster. Multi-mode viscoelastic simulations of cable-coating flows. under review in JNNFM, 1997/8.

[22] E.O.A. Carew, P. Townsend, and M.F. Webster. A Taylor-Petrov-Galerkin algorithm for viscoelastic flow. J. Non-Newtonian Fluid Mech., 50:253-287, 1993.

[23] D.M Hawken, P. Townsend, and M.F. Webster. A comparison of gradient recovery methods in finite element calculations. Commun. Appl. Numer. Meth., 7:195-204, 1991.

[24] O.C. Zienkiewicz and J.Z. Zhu. Superconvergence and the superconvergent patch recovery. Finite Element in Analysis and Design, 19:11-23, 1995.

[25] N. Phan-Thien. A non-linear network viscoelastic model. J. Rheol., 22:259-283, 1978.

[26] A.R. Davies, K. Walters, and D.M. Binding. An analysis of the discrete relaxation spectra of 3 polyethylene samples for BICC group, private communications. Technical report, Institute of non-Newtonian Fluid Mechanics, University of Wales, Aberystwyth, June 20, 1996.

[27] K. Walters, D.M. Binding, and R.E. Evans. Modelling the rheometric behaviour of 3 polyethylene melts. Technical report, Institute of non-Newtonian Fluid Mechanics, University of Wales, Aberystwyth, May 10, 1994.

[28] D.M. Hawken, H.R. Tamaddon-Jahromi, P. Townsend, and M.F. Webster. A TaylorGalerkin based algorithm for viscous incompressible flow. Int. J. Num. Meth. Fluids, 10:327-351, 1990 .

[29] P. Townsend and M.F. Webster. An algorithm for the three-dimensional transient simulation of non-Newtonian fluid flows. Proc. NUMETA 87, Martinus Nijhoff, Publishers, Dordrecht, 2:T12/1, 1987.

[30] E.O. Carew, P. Townsend, and M.F. Webster. A Taylor-Petrov-Galerkin algorithm for viscoelastic flow. J. Non-Newtonian Fluid Mech., 50:253-287, 1993.

[31] C. Cuvelier, A. Segal, and A.A. van Steenhoven. Finite Element Methods and NavierStokes Equations. D. Reidel Publishing Company, Holland, 1986.

[32] D.M. Binding. An approximate analysis for contraction and converging flows. J. NonNewtonian Fluid Mech., 27:173-189, 1988. 


\section{List of Tables}

1 Geometric dimensions. . . . . . . . . . . . . . . . . . . . 16

2 Three-mode model parameters. . . . . . . . . . . . . . . . 16

3 Seven-mode model parameters. . . . . . . . . . . . . . . . . 16

4 Mesh characteristics per region. . . . . . . . . . . . . . . . 16

5 Directional element distributions per region. . . . . . . . . . . . . . . . 17

\section{List of Figures}

1 Schematic diagram for tube-tooling domain: geometric adjustment. . . . . . . 18

2 Finite element mesh. . . . . . . . . . . . . . . . . . 19

3 HDPE polymer-PTT fits, comparison of three and seven-mode models, $\epsilon=1$,

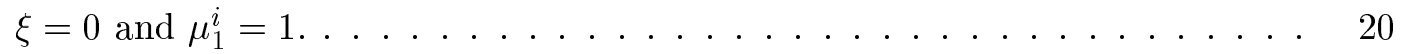

4 Streamwise profiles, $\tau_{r z}$ and $\tau_{z z}$ for Case a, b and c, along inner wall. . . . . 21

5 Streamwise profiles, $\tau_{r z}$ and $\tau_{z z}$ for Case a, e and f, along inner wall. . . . . . 22

6 Streamwise profiles, $\tau_{z z}$ for Case a-f, at centreline. . . . . . . . . . . . . 23

$7 \quad$ Streamwise profiles, $\tau_{r z}$ for Case a-f, at centreline. . . . . . . . . . . . . . 24

8 Streamwise profiles, $\tau_{r z}$ and $\tau_{z z}$ for Case a, b and e, along inner coating surface. 25

9 Streamwise profiles, $\tau_{r z}$ and $\tau_{z z}$ for Case a, for different modes along inner wall. 26

10 Streamwise profiles, $\tau_{r z}$ and $\tau_{z z}$ for Case $\mathrm{b}$, for different modes along inner wall. 27

11 Streamwise profiles, $\tau_{r z}$ and $\tau_{z z}$ for Case e, for different modes along inner wall. 28

12 Streamwise profiles, $\tau_{z z}$ for Case a, and fully developed inlet land flow, at centreline and along inner coating/die wall surface. . . . . . . . . . . . . 29

13 Streamwise profiles, $\tau_{r z}$ and $\tau_{z z}$ for Case a, three and seven-mode models along inner wall. ............................ 30

14 Streamwise profiles, $\tau_{z z}$, seven-mode models, comparison of individual mode

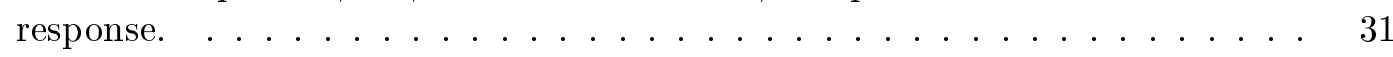




\begin{tabular}{|cccccccccccc|}
\hline \hline Cases & $L_{1}$ & $L_{2}$ & $L_{3}$ & $L_{4}$ & $L_{5}$ & $R_{1}$ & $R_{2}$ & $R_{3}$ & $R_{4}$ & $R_{5}$ & $R_{6}$ \\
\hline \hline a) & 0.83 & 0.83 & 0.17 & 1.00 & 0.50 & 0.68 & 0.77 & 0.18 & 0.23 & 0.13 & 0.17 \\
\hline b) & 0.83 & 0.83 & 0.17 & 0.50 & 0.50 & 0.68 & 0.77 & 0.18 & 0.23 & 0.13 & 0.17 \\
\hline c) & 0.83 & 0.42 & 0.17 & 1.00 & 0.50 & 0.68 & 0.77 & 0.18 & 0.23 & 0.13 & 0.17 \\
\hline d) & 0.83 & 0.42 & 0.17 & 0.50 & 0.50 & 0.68 & 0.77 & 0.18 & 0.23 & 0.13 & 0.17 \\
\hline e) & 0.83 & 0.83 & 0.17 & 1.00 & 0.50 & 0.68 & 0.77 & 0.15 & 0.20 & 0.13 & 0.17 \\
\hline f) & 0.83 & 0.83 & 0.17 & 1.00 & 0.50 & 0.58 & 0.77 & 0.15 & 0.20 & 0.13 & 0.17 \\
\hline
\end{tabular}

Table 1: Geometric dimensions.

\begin{tabular}{|cccc|}
\hline \hline$\lambda_{i}(s)$ & $\mu_{i}($ Pa.s $)$ & $g_{i}=\frac{\mu_{i}}{\lambda_{i}}$ & $W_{e}^{i}$ \\
\hline \hline 0.017 & 2792 & 164222 & 0.095 \\
\hline 0.87 & 19595 & 22523 & 4.84 \\
\hline 59.57 & 116797 & 1961 & 331.5 \\
\hline
\end{tabular}

Table 2: Three-mode model parameters.

\begin{tabular}{|cccc|}
\hline \hline$\lambda_{i}(s)$ & $\mu_{i}($ Pa.s $)$ & $g_{i}=\frac{\mu_{i}}{\lambda_{i}}$ & $W_{e}^{i}$ \\
\hline \hline $3.79 e^{-3}$ & 817.0 & 215713 & $2.11 e^{-2}$ \\
\hline $3.89 e^{-2}$ & 2423.0 & 62217 & 0.22 \\
\hline 0.29 & 6452.0 & 22044 & 1.62 \\
\hline 2.27 & 15235.0 & 6716 & 12.6 \\
\hline 18.19 & 34636.0 & 1903 & 101.3 \\
\hline 226.8 & 170736.0 & 753 & 1262 \\
\hline 1345.7 & 6371.0 & 5 & 7490 \\
\hline
\end{tabular}

Table 3: Seven-mode model parameters.

\begin{tabular}{|ccccccc|}
\hline \hline $\begin{array}{c}\text { Cases } \\
\text { Sections }\end{array}$ & a) & b) & $\begin{array}{c}\text { c) } \\
\text { elements }\end{array}$ & d) & e) & f) \\
\hline \hline Inlet tube & 680 & 680 & 680 & 680 & 680 & 680 \\
\hline Converging section & 680 & 680 & 380 & 380 & 680 & 680 \\
\hline Land region & 120 & 120 & 160 & 160 & 120 & 120 \\
\hline Draw-down region & 800 & 400 & 800 & 400 & 800 & 800 \\
\hline wire region & 800 & 800 & 800 & 800 & 800 & 800 \\
\hline Total & 3080 & 2680 & 2820 & 2420 & 3080 & 3080 \\
\hline
\end{tabular}

Table 4: Mesh characteristics per region. 


\begin{tabular}{|c|ccccc|}
\hline \hline & $\begin{array}{c}\text { inlet } \\
\text { tube }\end{array}$ & $\begin{array}{c}\text { converging } \\
\text { tube }\end{array}$ & $\begin{array}{c}\text { land } \\
\text { region }\end{array}$ & $\begin{array}{c}\text { draw } \\
\text { down }\end{array}$ & $\begin{array}{c}\text { along } \\
\text { wire }\end{array}$ \\
\hline \hline Axial & 34 & 34 & 6 & 40 & 20 \\
---- & ---- & ---- & ---- & ---- & ---- \\
Radial & 10 & 10 & 10 & 10 & 10 \\
\hline
\end{tabular}

Table 5: Directional element distributions per region. 
a)

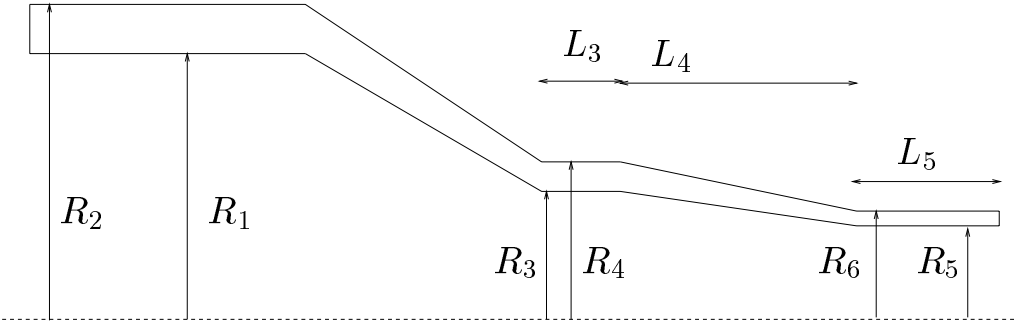

b)
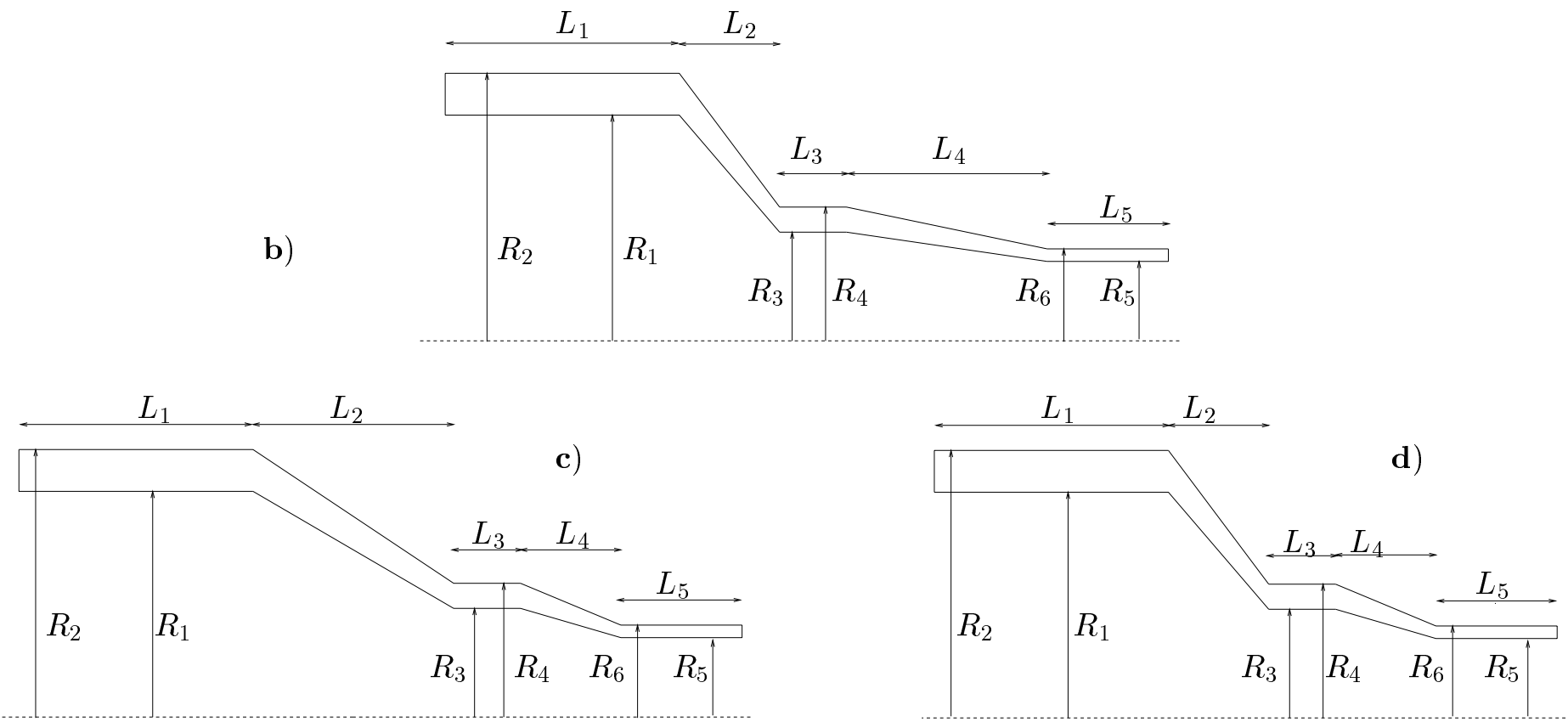

e)

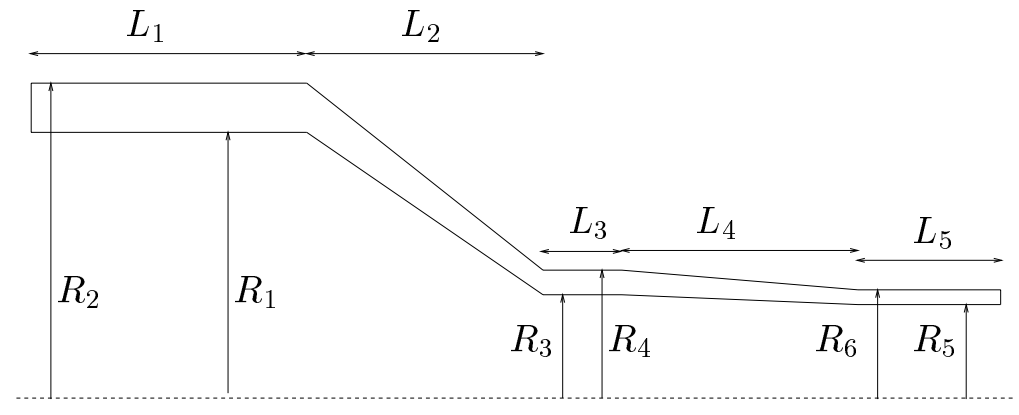

f)

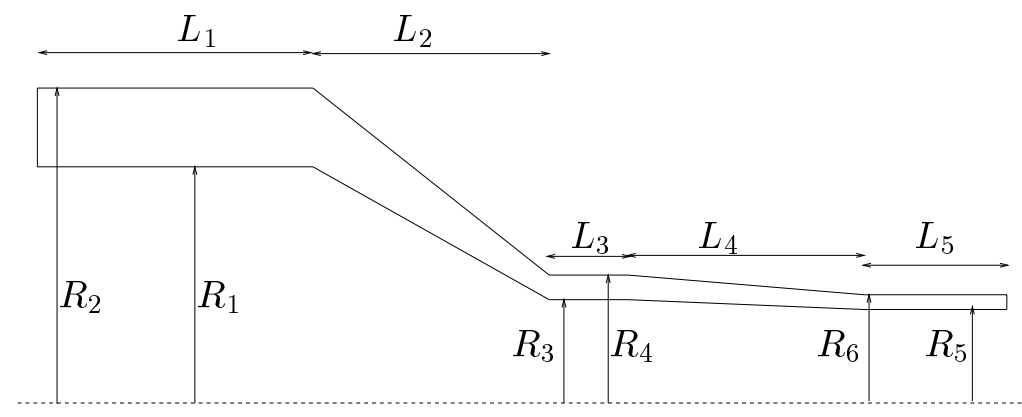

Figure 1: Schematic diagram for tube-tooling domain: geometric adjustment. 


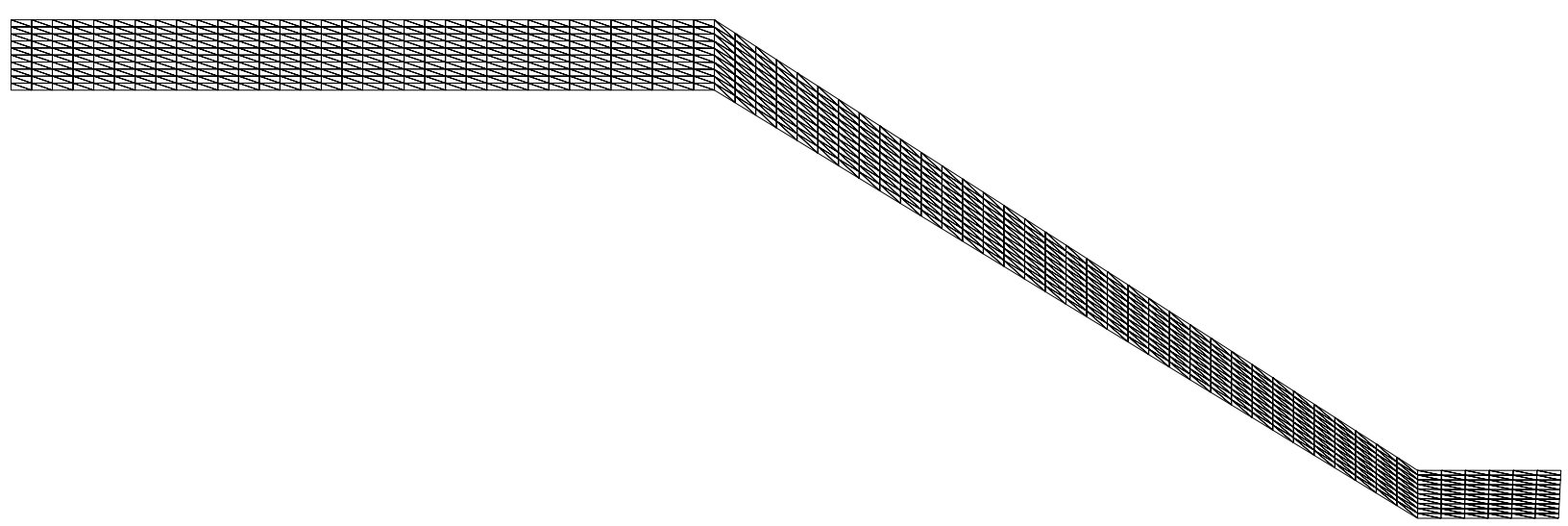

Die section

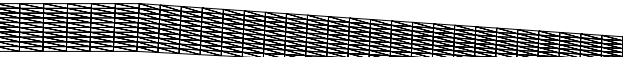

\section{Draw-down section}

Figure 2: Finite element mesh. 


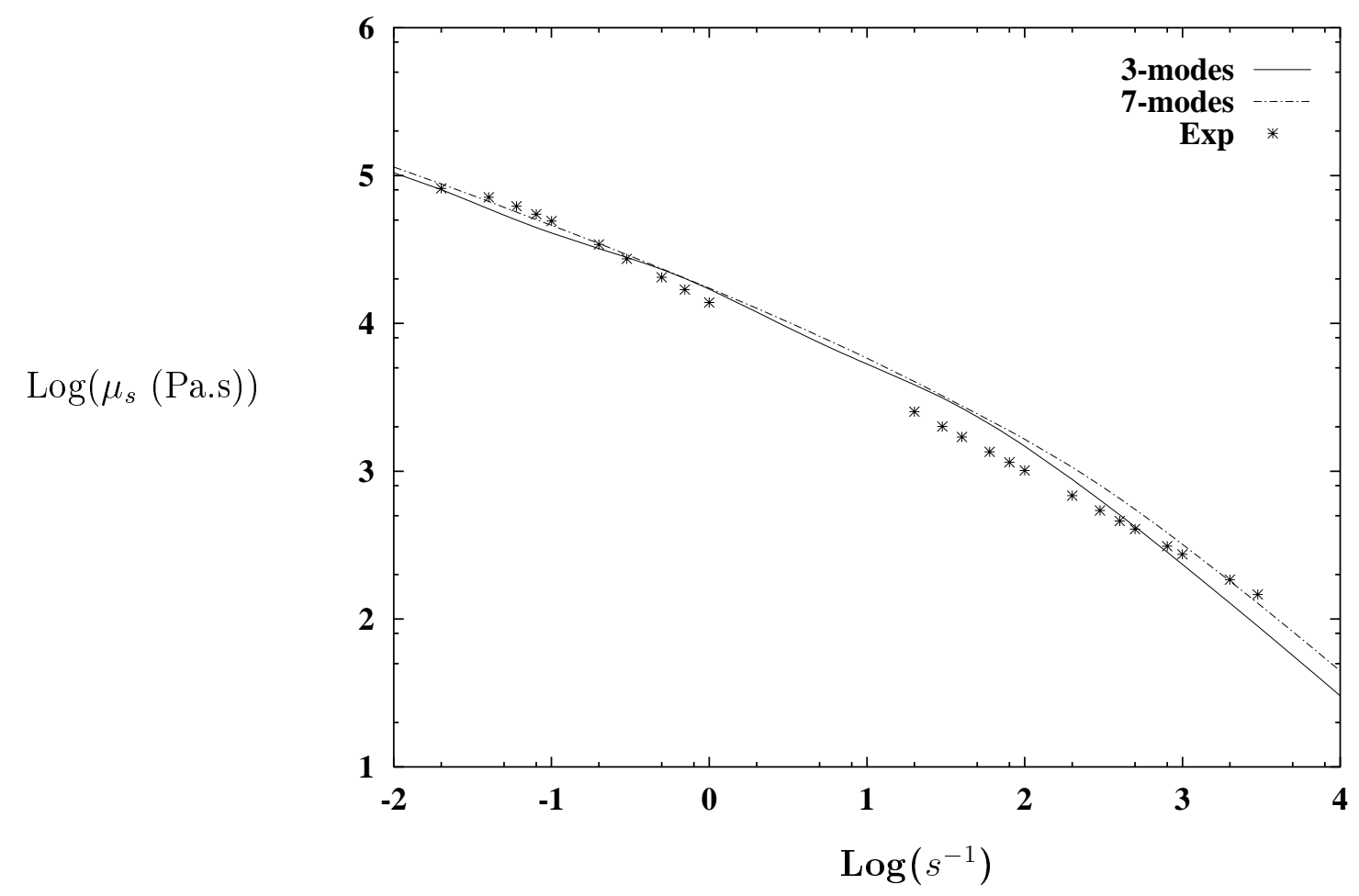

a) $\mu_{s}$ fits

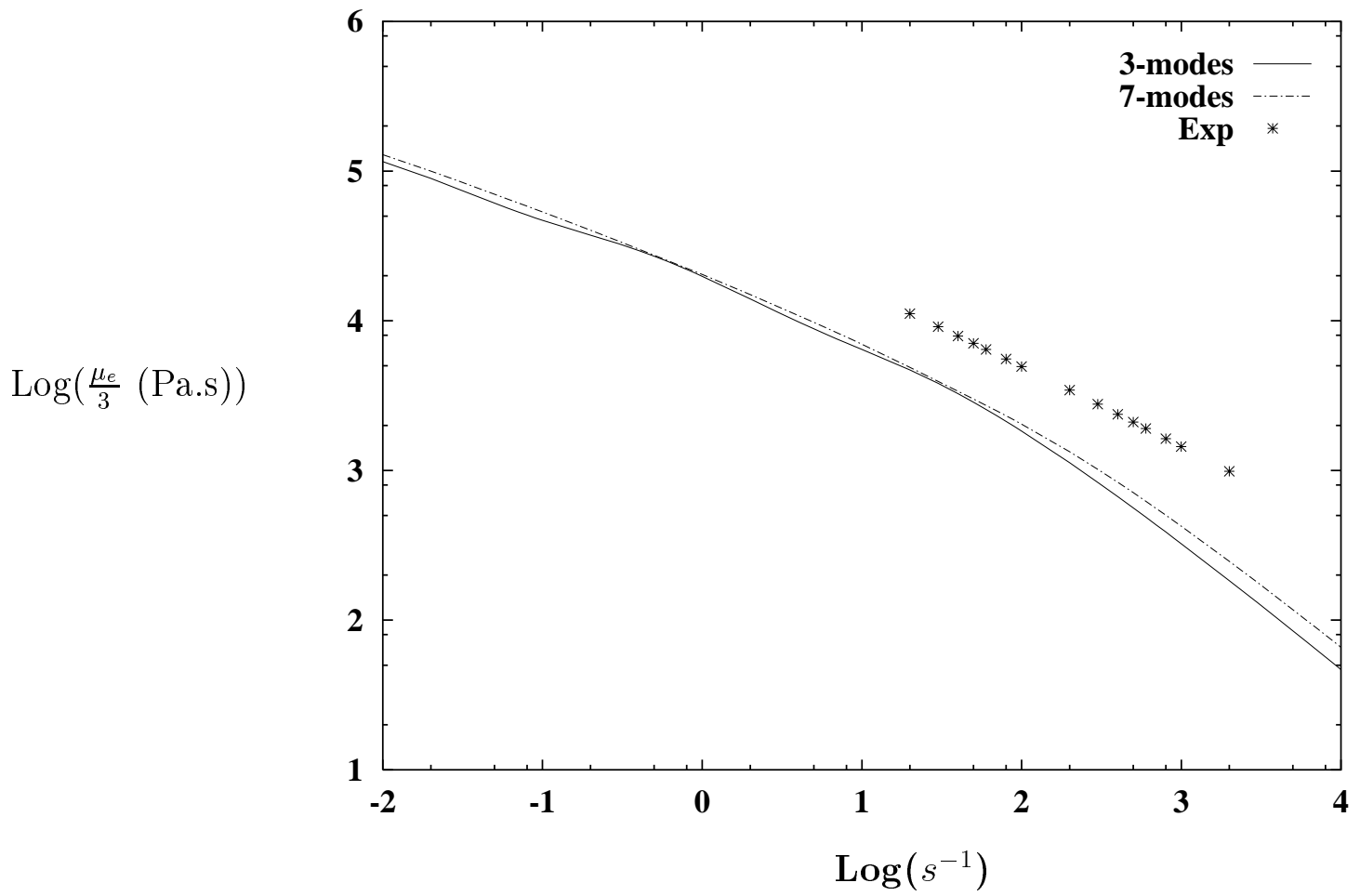

b) $\mu_{e}$ fits

Figure 3: HDPE polymer-PTT fits, comparison of three and seven-mode models, $\epsilon=1, \xi=0$ and $\mu_{1}^{i}=1$. 


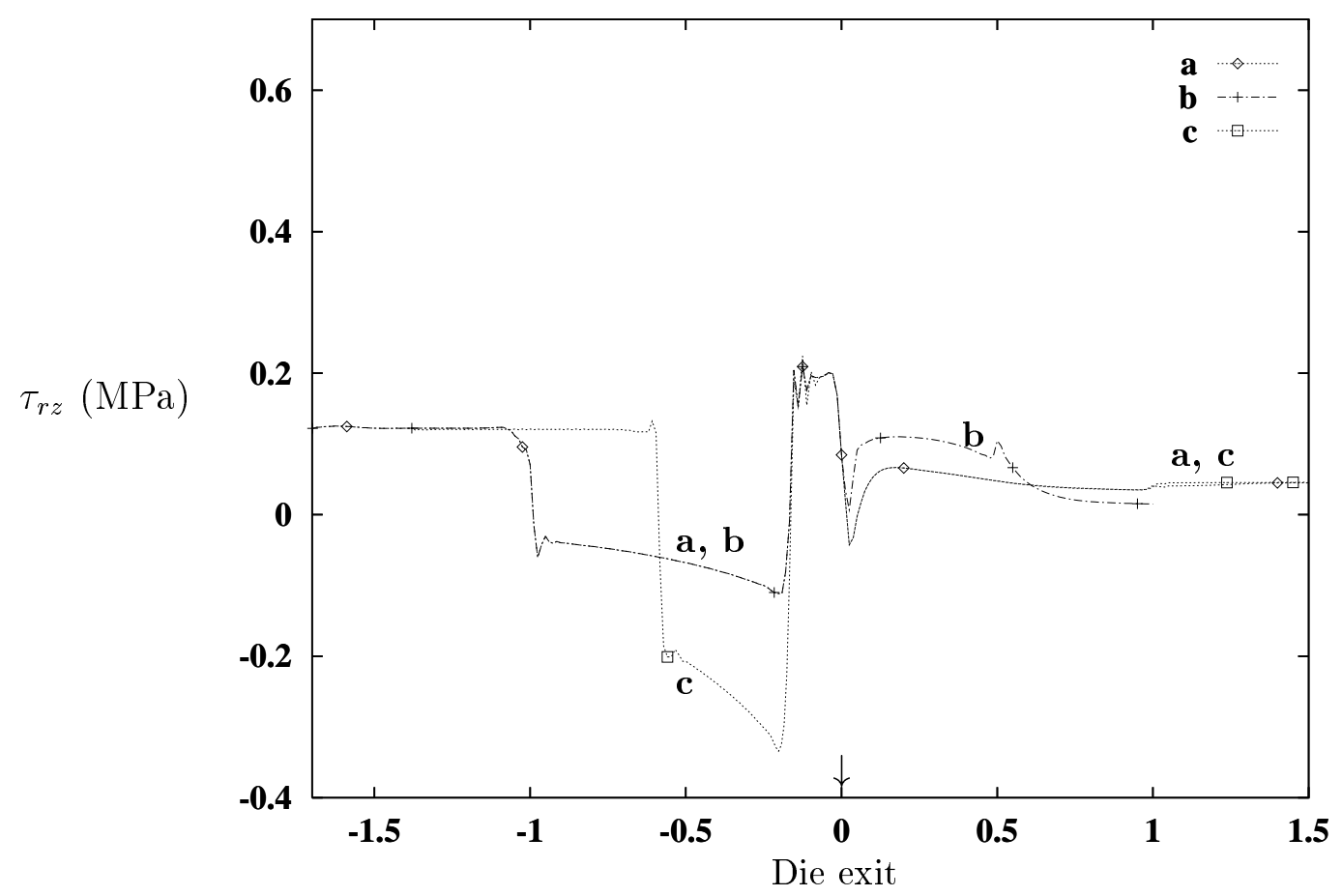

Axial direction, $\mathrm{z}$

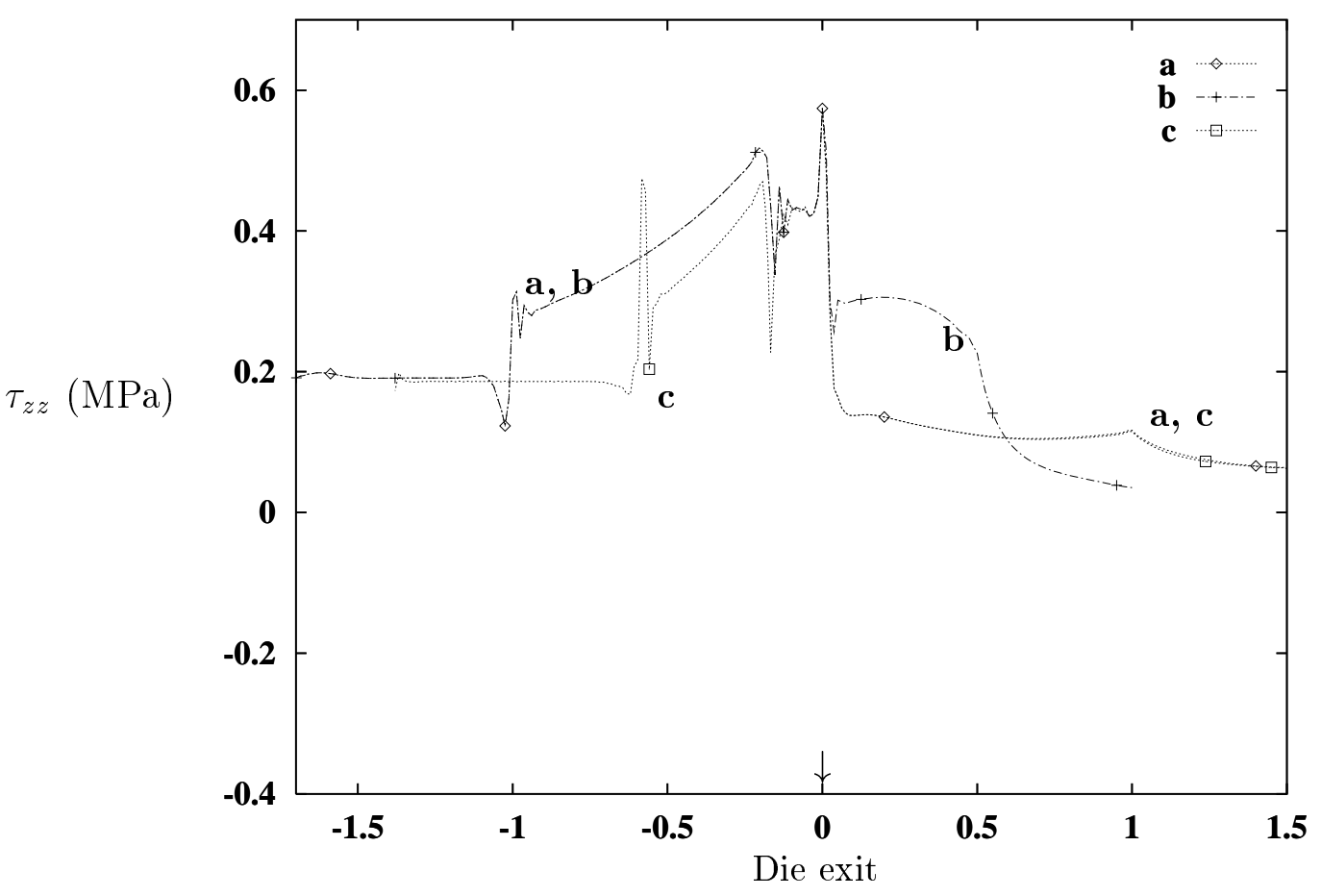

Axial direction, $\mathrm{z}$

Figure 4: Streamwise profiles, $\tau_{r z}$ and $\tau_{z z}$ for Case a, b and c, along inner wall. 


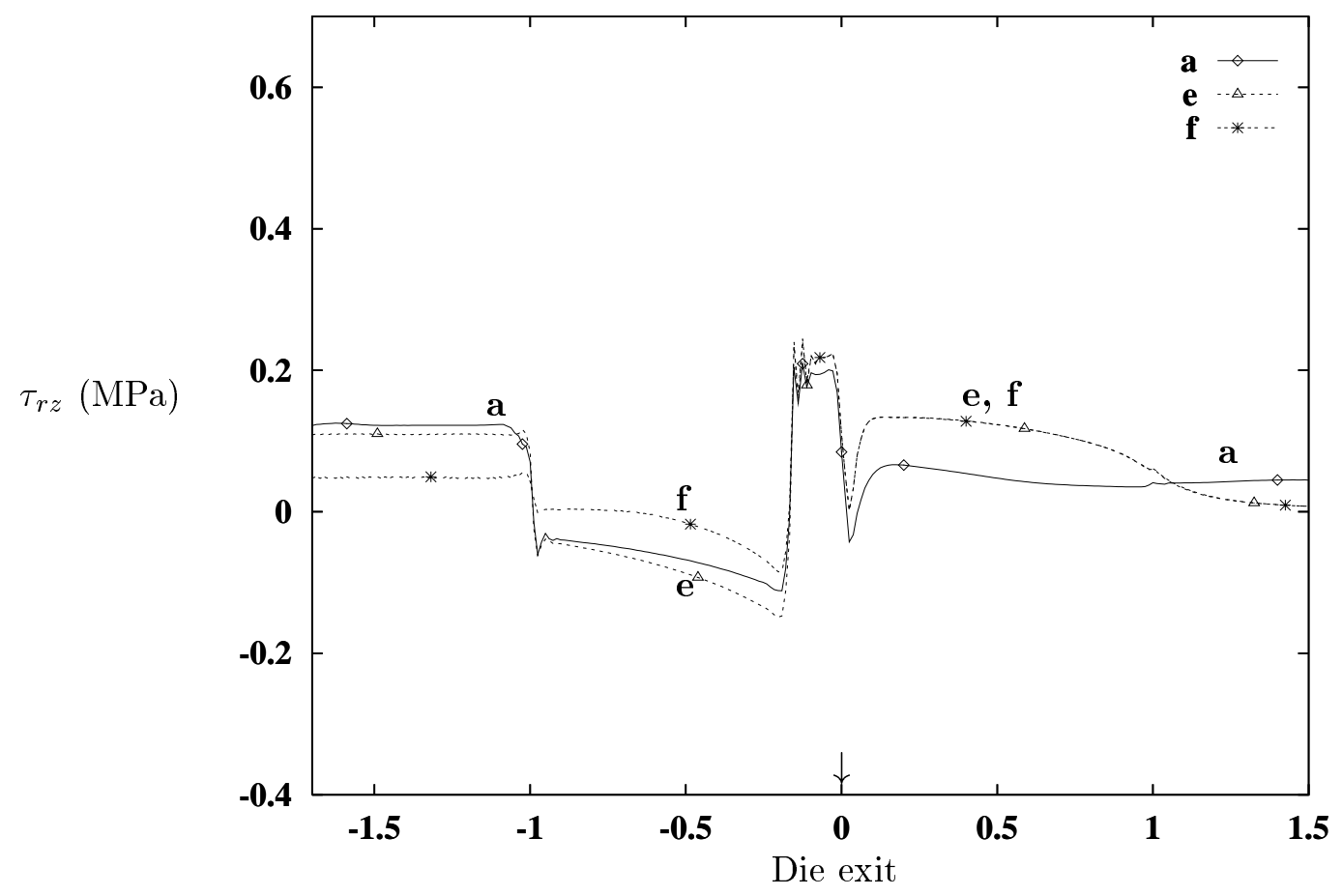

Axial direction, $\mathrm{z}$

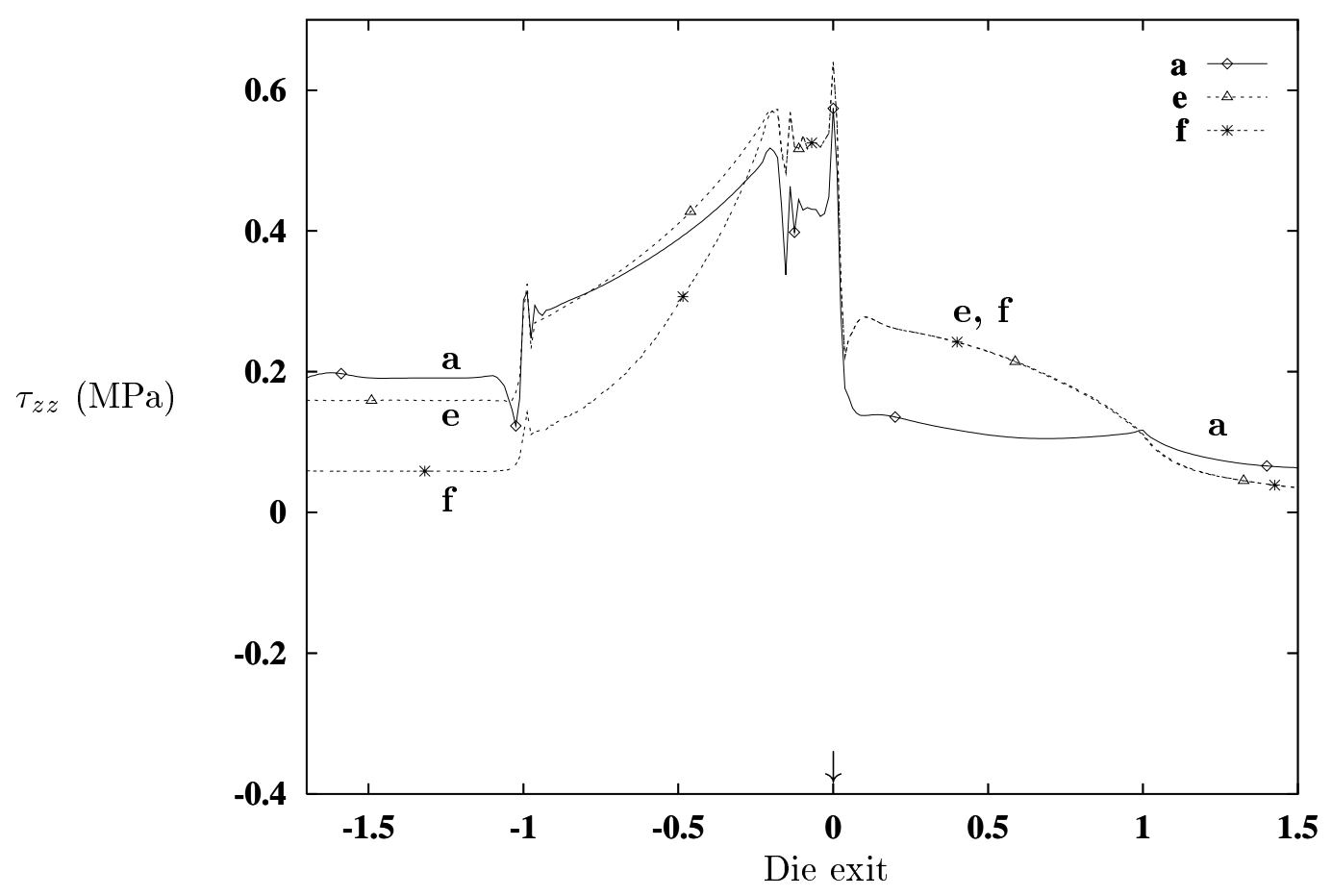

Axial direction, $\mathrm{z}$

Figure 5: Streamwise profiles, $\tau_{r z}$ and $\tau_{z z}$ for Case a, e and f, along inner wall. 


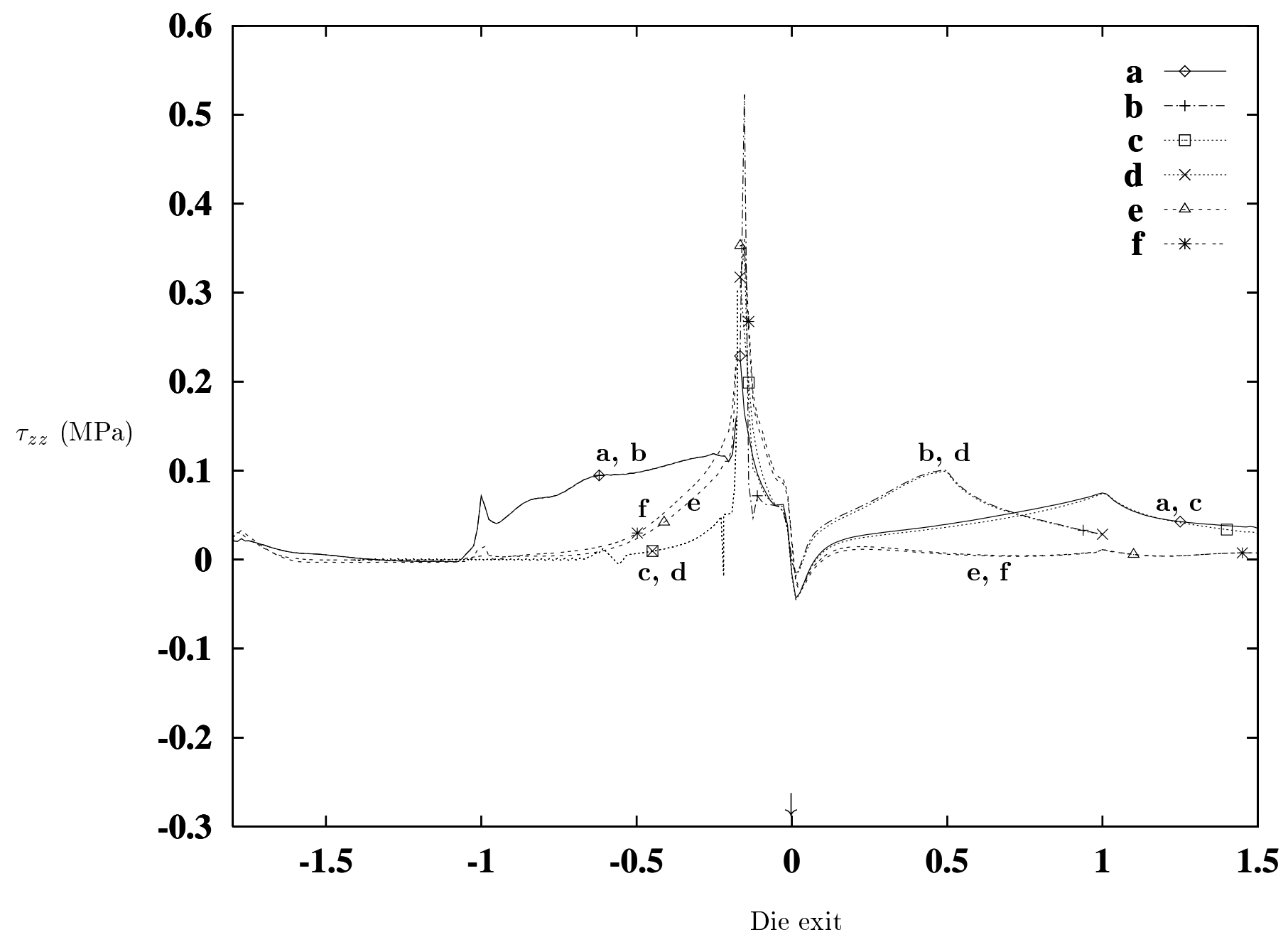

Axial direction, $\mathrm{z}$

Figure 6: Streamwise profiles, $\tau_{z z}$ for Case a-f, at centreline. 


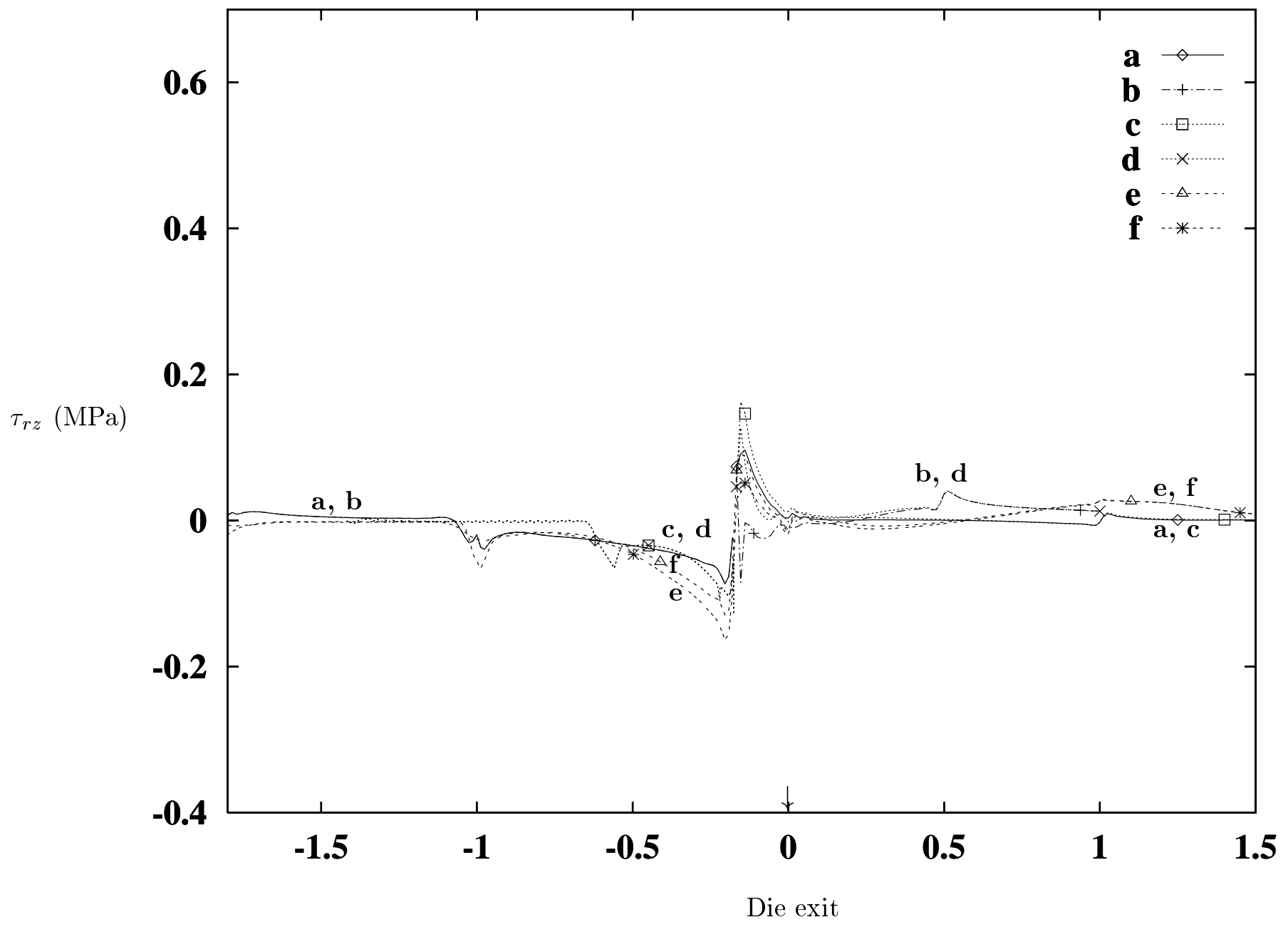

Axial direction, $\mathrm{z}$

Figure 7: Streamwise profiles, $\tau_{r z}$ for Case a-f, at centreline. 


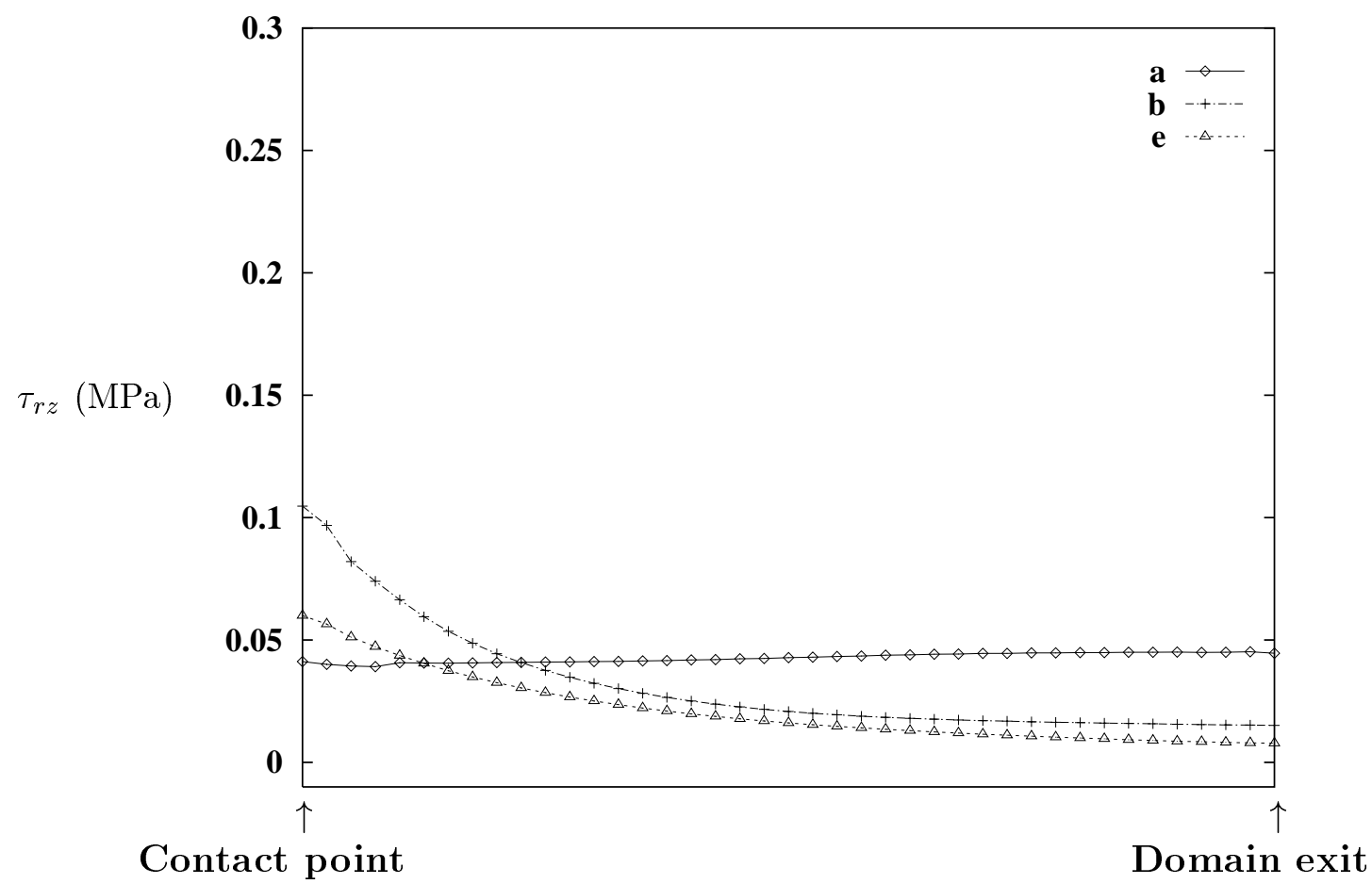

Axial direction, $\mathrm{z}$

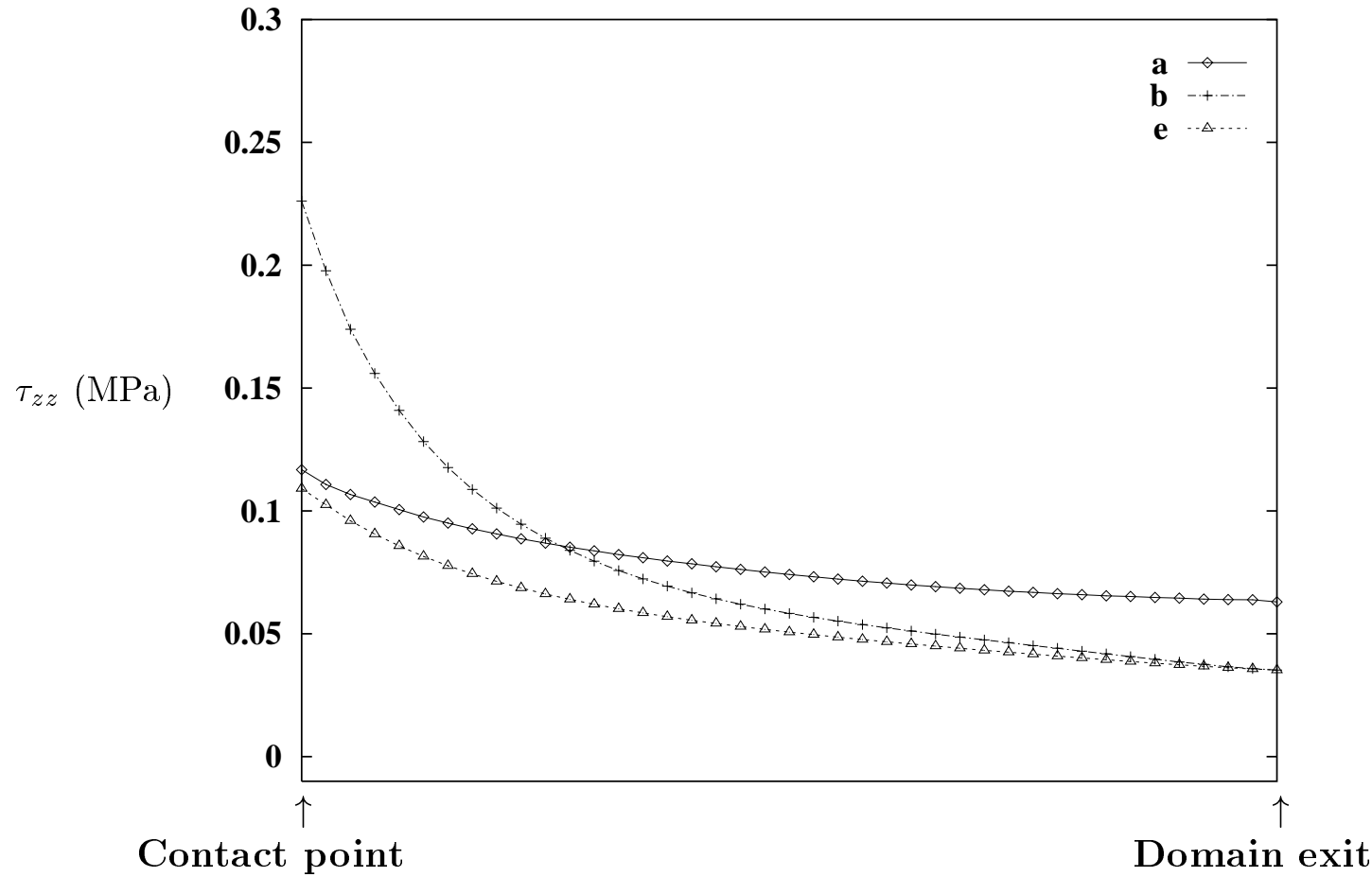

Axial direction, $\mathrm{z}$

Figure 8: Streamwise profiles, $\tau_{r z}$ and $\tau_{z z}$ for Case a, b and e, along inner coating surface. 

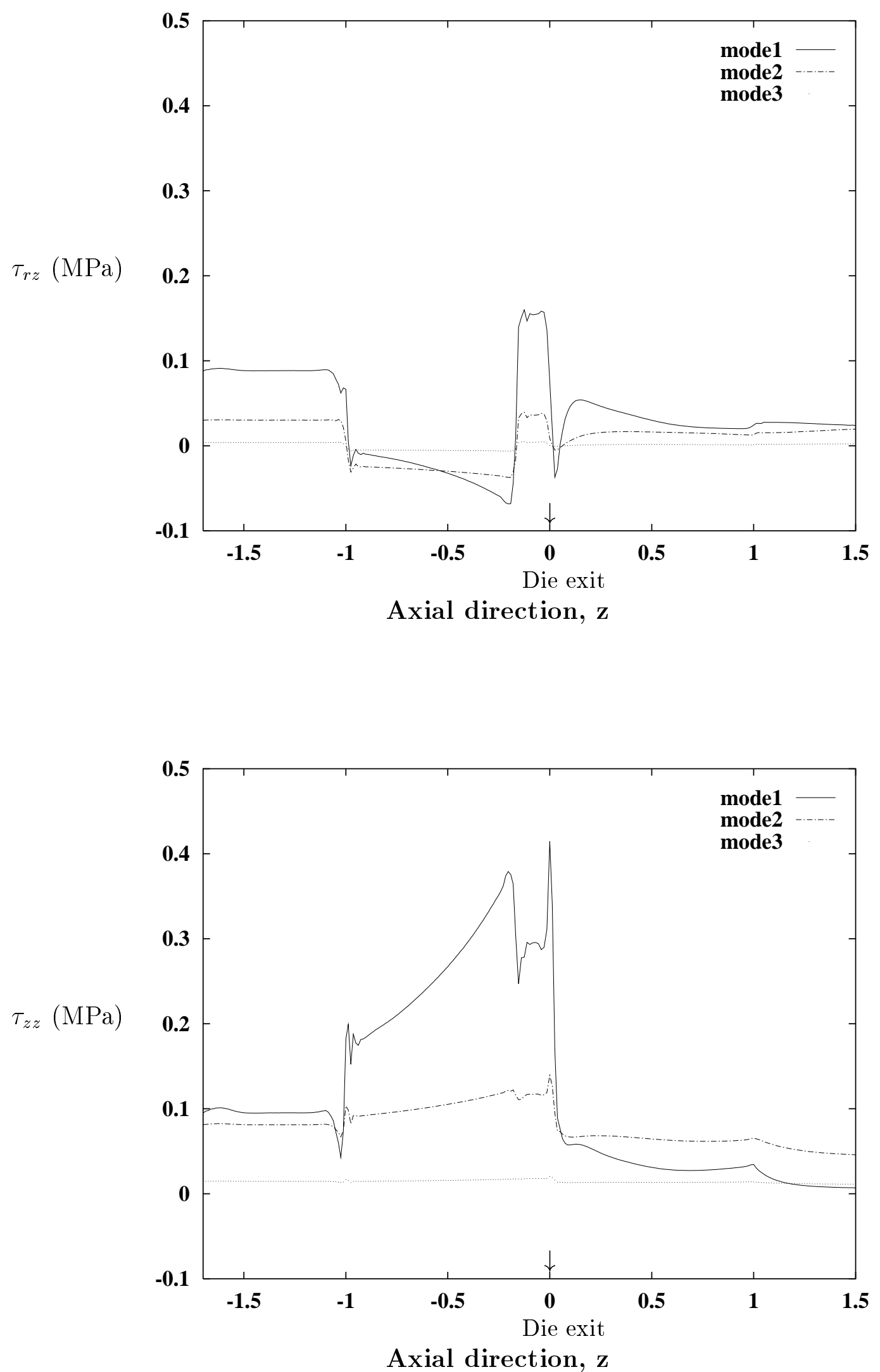

Figure 9: Streamwise profiles, $\tau_{r z}$ and $\tau_{z z}$ for Case a, for different modes along inner wall. 


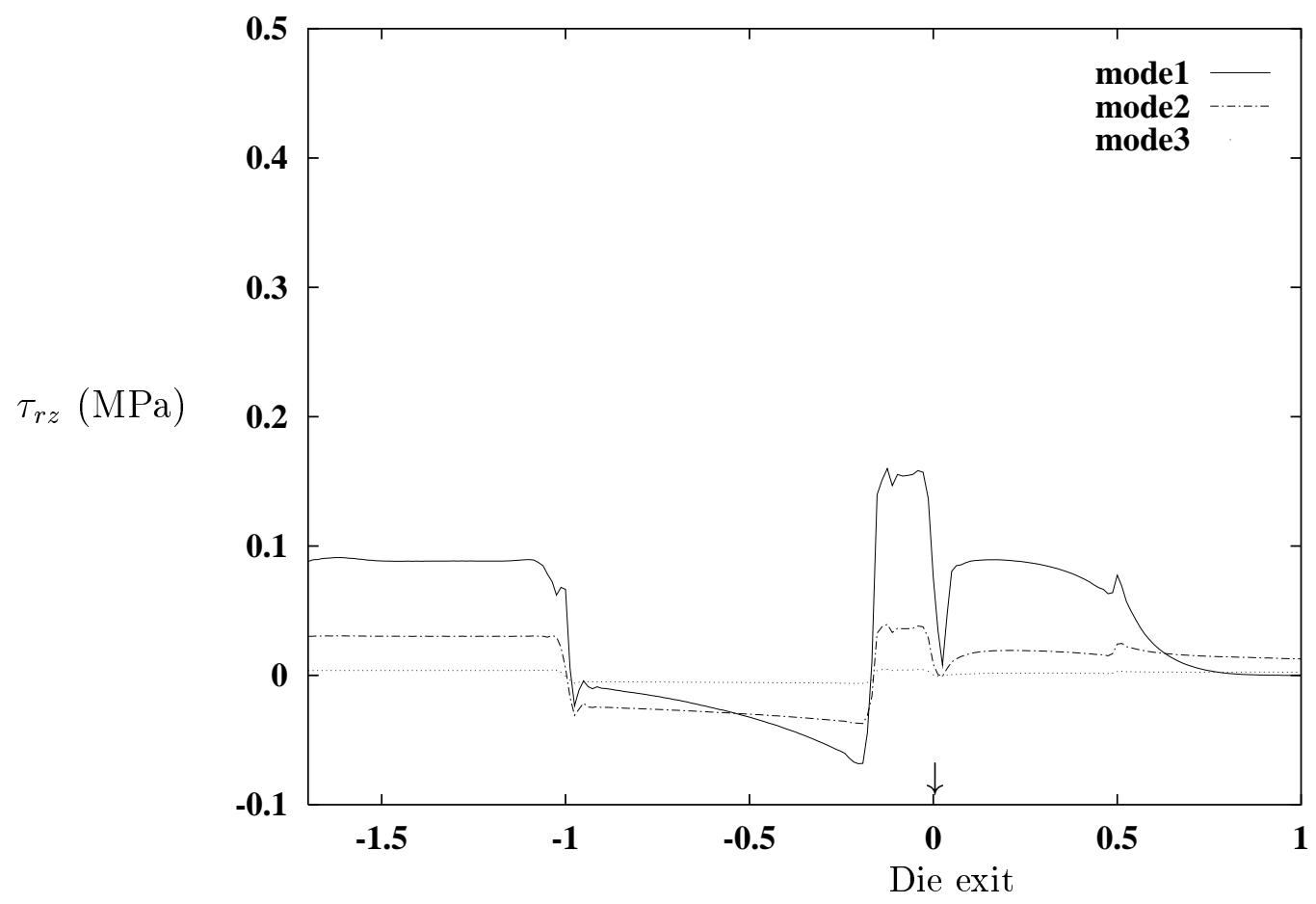

Axial direction, $\mathrm{z}$

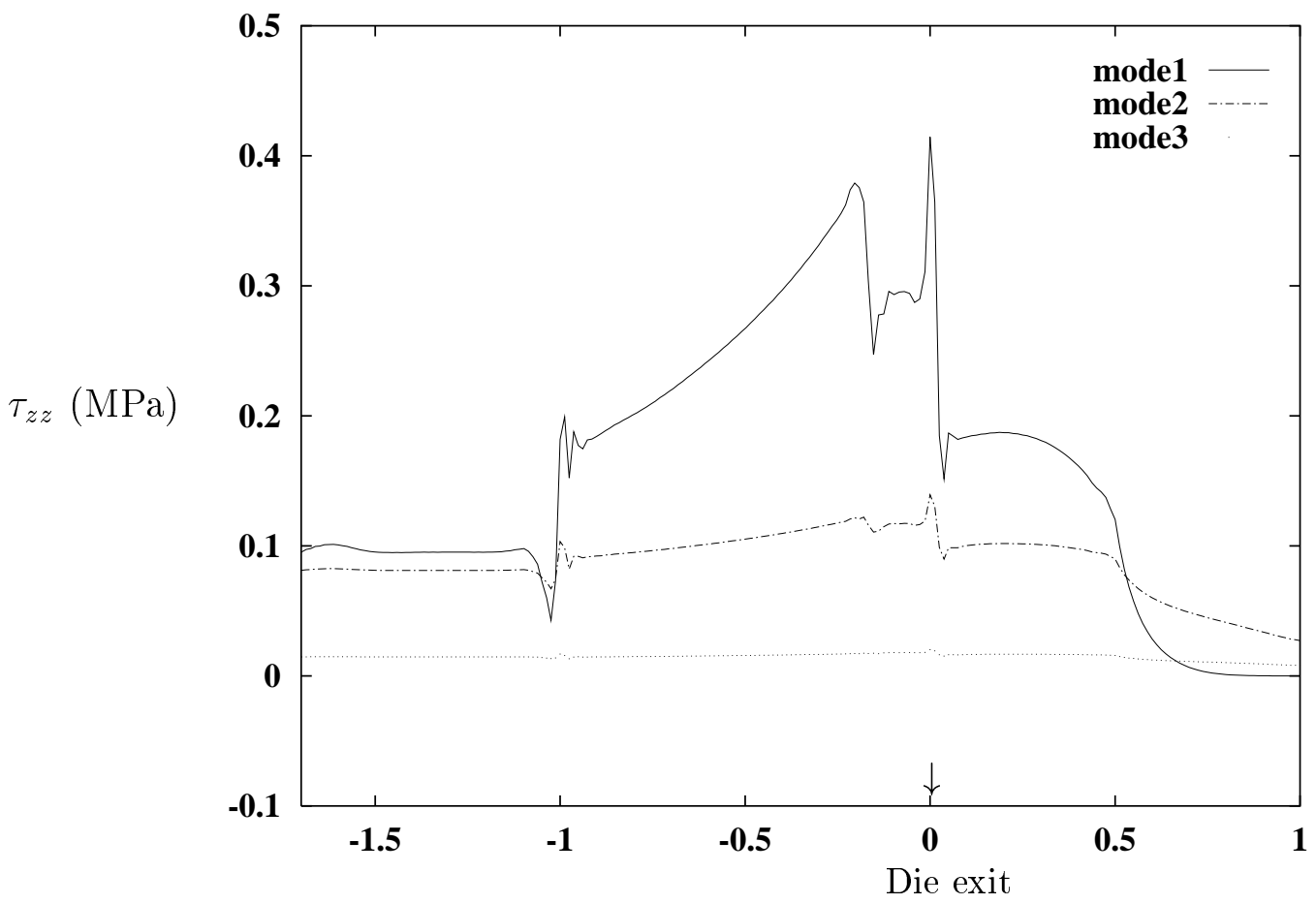

Axial direction, $\mathrm{z}$

Figure 10: Streamwise profiles, $\tau_{r z}$ and $\tau_{z z}$ for Case $\mathrm{b}$, for different modes along inner wall. 

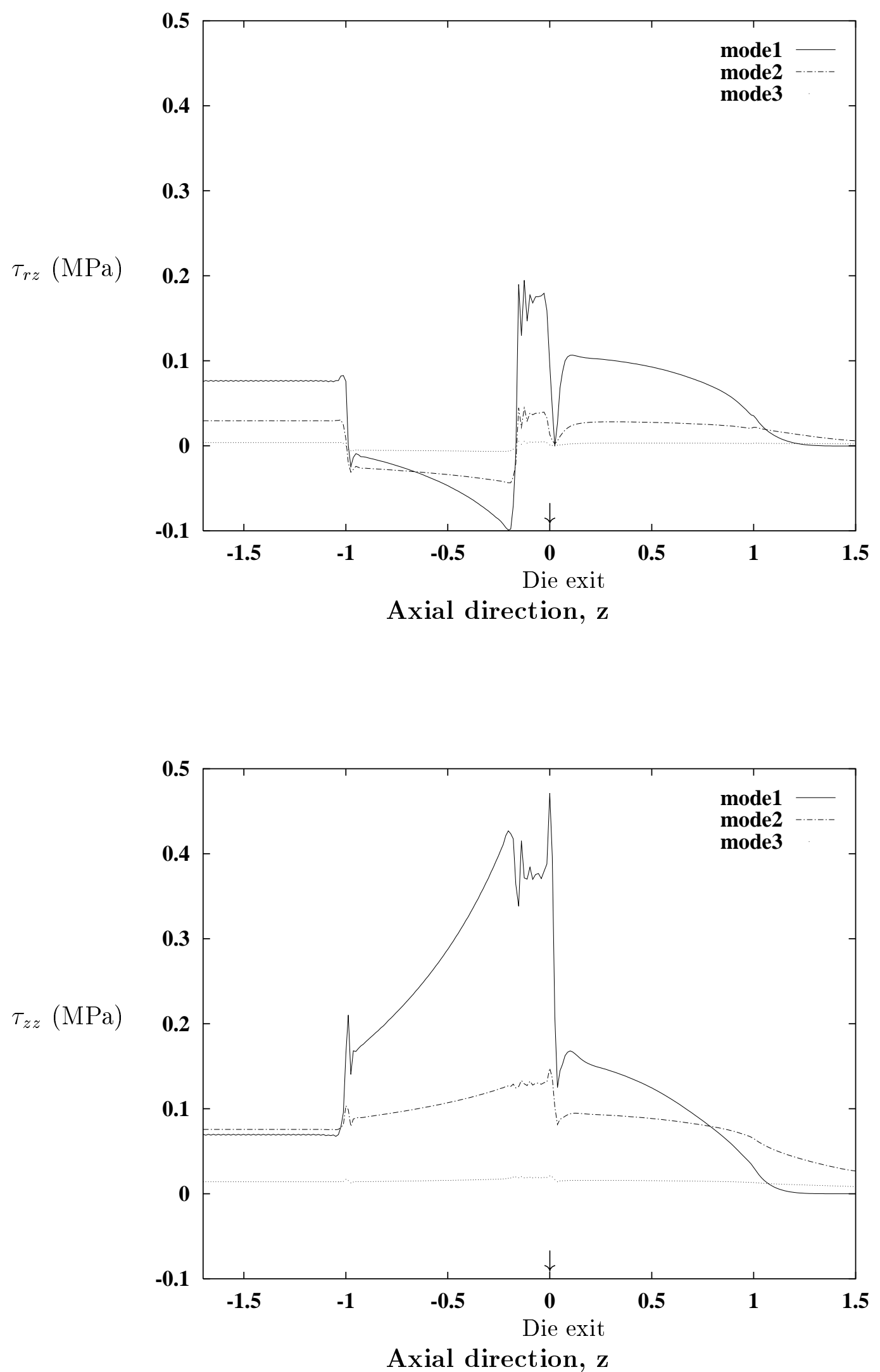

Figure 11: Streamwise profiles, $\tau_{r z}$ and $\tau_{z z}$ for Case e, for different modes along inner wall. 


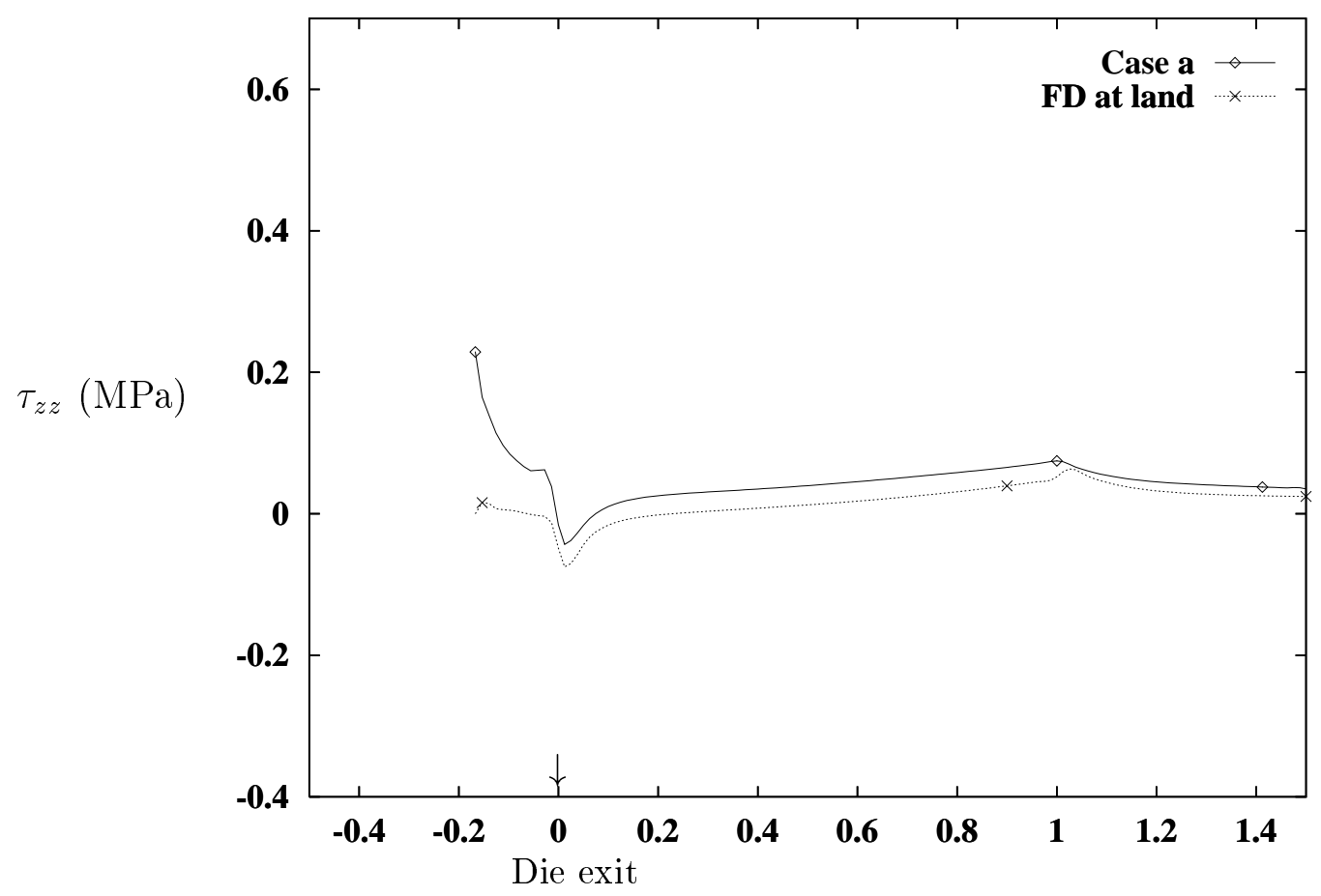

Axial direction, $\mathrm{z}$

a) at centreline

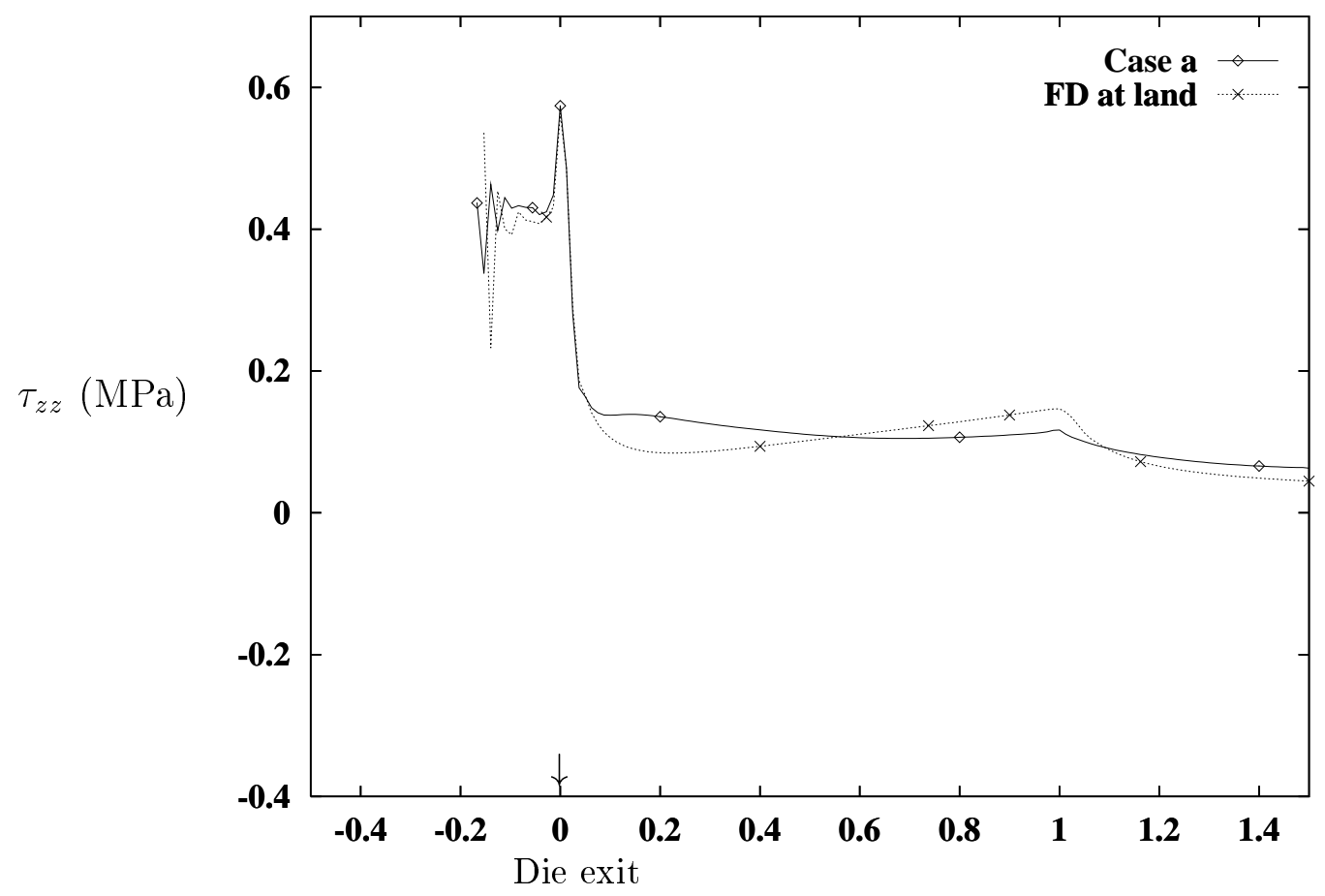

Axial direction, $\mathrm{z}$

b) along inner coating/die wall surface

Figure 12: Streamwise profiles, $\tau_{z z}$ for Case a, and fully developed inlet land flow, at centreline and along inner coating/die wall surface. 


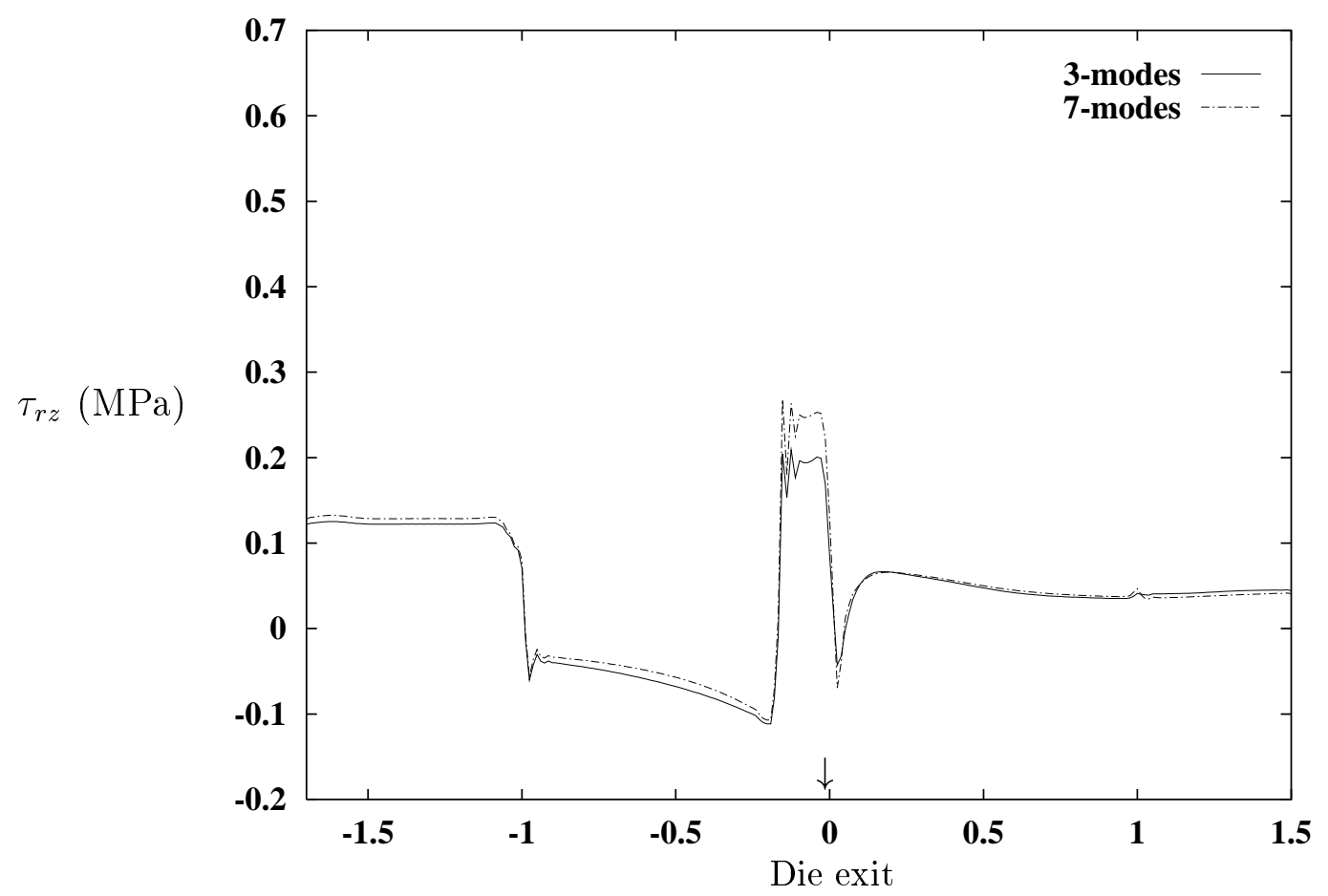

Axial direction, $\mathrm{z}$

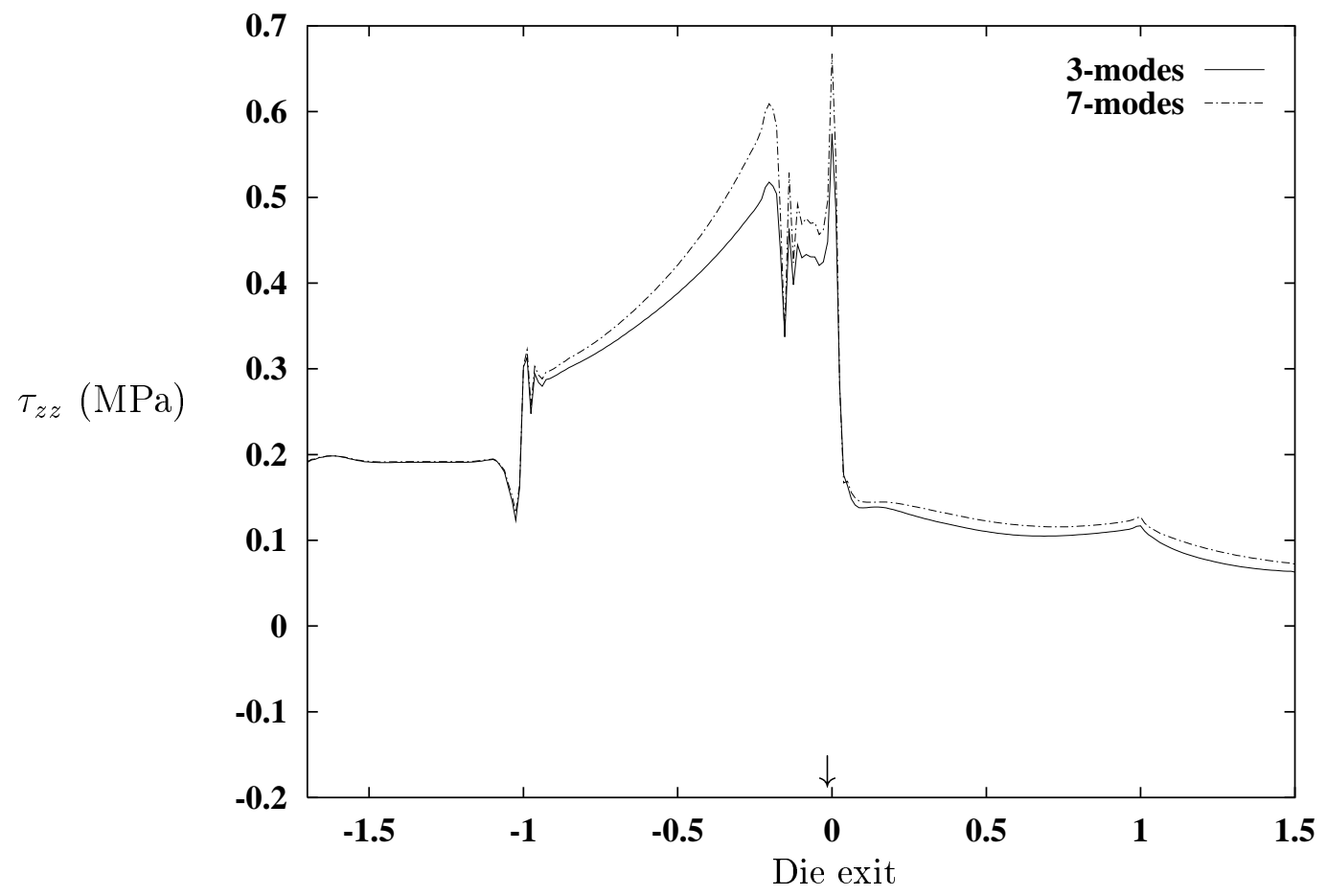

Axial direction, $\mathrm{z}$

Figure 13: Streamwise profiles, $\tau_{r z}$ and $\tau_{z z}$ for Case a, three and seven-mode models along inner wall. 


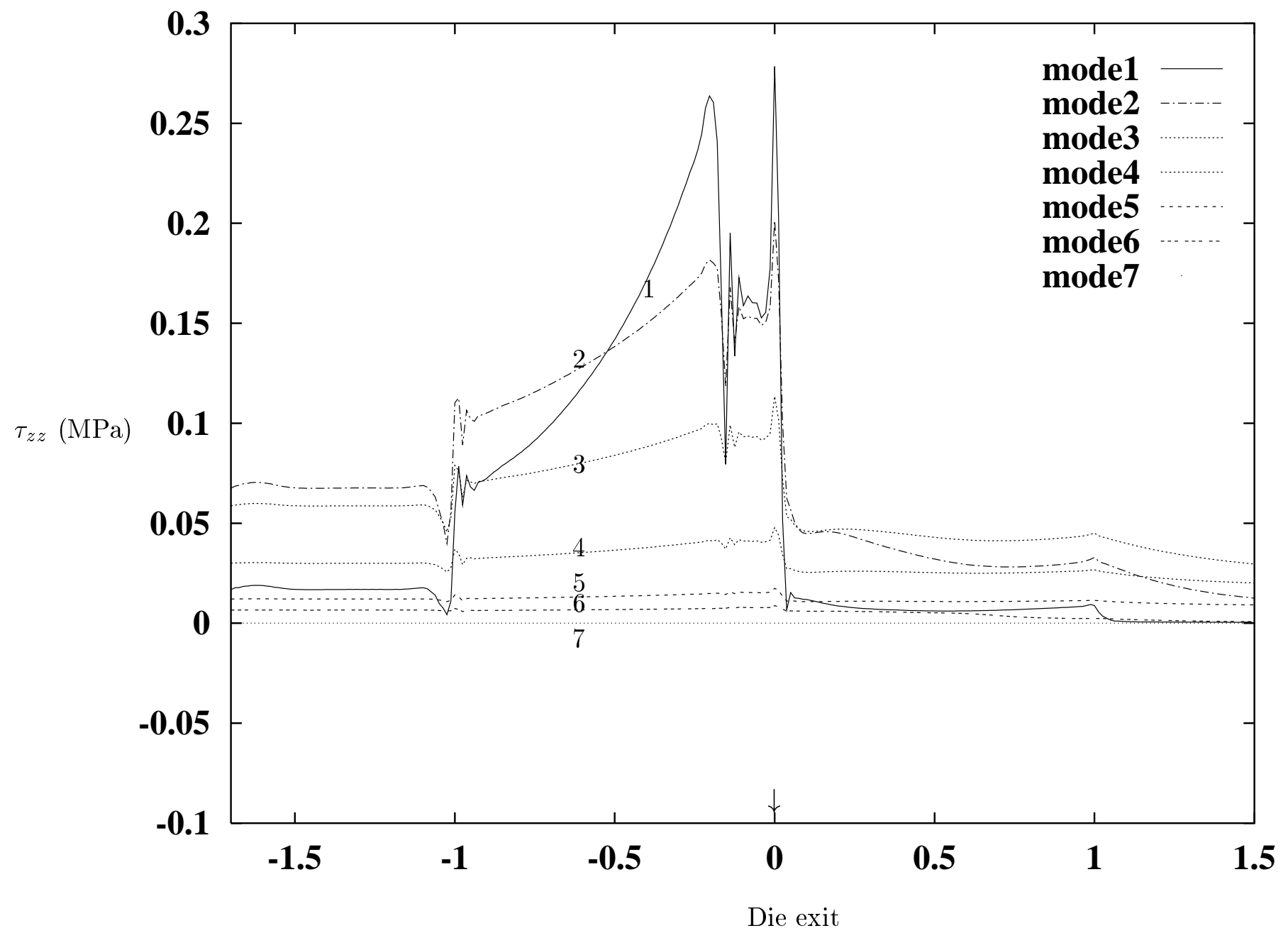

Axial direction, $\mathrm{z}$

Figure 14: Streamwise profiles, $\tau_{z z}$, seven-mode models, comparison of individual mode response. 